\title{
A Blockchain Framework for Patient-Centered Health Records and Exchange (HealthChain): Evaluation and Proof-of-Concept Study
}

\author{
Ray Hales Hylock, PhD; Xiaoming Zeng, MD, PhD \\ Department of Health Services and Information Management, College of Allied Health Sciences, East Carolina University, Greenville, NC, United \\ States
}

\section{Corresponding Author:}

Ray Hales Hylock, PhD

Department of Health Services and Information Management

College of Allied Health Sciences

East Carolina University

4340 Health Sciences Building, Mail Stop 668

Greenville, NC, 27858

United States

Phone: 12527446184

Email: hylockr@ecu.edu

\begin{abstract}
Background: Blockchain has the potential to disrupt the current modes of patient data access, accumulation, contribution, exchange, and control. Using interoperability standards, smart contracts, and cryptographic identities, patients can securely exchange data with providers and regulate access. The resulting comprehensive, longitudinal medical records can significantly improve the cost and quality of patient care for individuals and populations alike.

Objective: This work presents HealthChain, a novel patient-centered blockchain framework. The intent is to bolster patient engagement, data curation, and regulated dissemination of accumulated information in a secure, interoperable environment. A mixed-block blockchain is proposed to support immutable logging and redactable patient blocks. Patient data are generated and exchanged through Health Level-7 Fast Healthcare Interoperability Resources, allowing seamless transfer with compliant systems. In addition, patients receive cryptographic identities in the form of public and private key pairs. Public keys are stored in the blockchain and are suitable for securing and verifying transactions. Furthermore, the envisaged system uses proxy re-encryption (PRE) to share information through revocable, smart contracts, ensuring the preservation of privacy and confidentiality. Finally, several PRE improvements are offered to enhance performance and security.
\end{abstract}

Methods: The framework was formulated to address key barriers to blockchain adoption in health care, namely, information security, interoperability, data integrity, identity validation, and scalability. It supports 16 configurations through the manipulation of 4 modes. An open-source, proof-of-concept tool was developed to evaluate the performance of the novel patient block components and system configurations. To demonstrate the utility of the proposed framework and evaluate resource consumption, extensive testing was performed on each of the 16 configurations over a variety of scenarios involving a variable number of existing and imported records.

Results: The results indicate several clear high-performing, low-bandwidth configurations, although they are not the strongest cryptographically. Of the strongest models, one's anticipated cumulative record size is shown to influence the selection. Although the most efficient algorithm is ultimately user specific, Advanced Encryption Standard-encrypted data with static keys, incremental server storage, and no additional server-side encryption are the fastest and least bandwidth intensive, whereas proxy re-encrypted data with dynamic keys, incremental server storage, and additional server-side encryption are the best performing of the strongest configurations.

Conclusions: Blockchain is a potent and viable technology for patient-centered access to and exchange of health information. By integrating a structured, interoperable design with patient-accumulated and generated data shared through smart contracts into a universally accessible blockchain, HealthChain presents patients and providers with access to consistent and comprehensive medical records. Challenges addressed include data security, interoperability, block storage, and patient-administered data access, with several configurations emerging for further consideration regarding speed and security. 
(J Med Internet Res 2019;21(8):e13592) doi: 10.2196/13592

\section{KEYWORDS}

blockchain; chameleon hashing; health information exchange; health information management; HL7 FHIR; patient-centered health; medical records; proxy re-encryption; redactable blockchain; smart contracts; digital health; electronic health records

\section{Introduction}

\section{Overview}

Health care is a data-intensive domain with vast amounts of information generated, accessed, and disseminated daily. Unfortunately, patient records are typically isolated in institution-centric electronic health records (EHRs), resulting in fragmentation with consequences ranging from inefficient care coordination to lack of critical information during emergencies [1-3]. Interoperability requirements were instituted as a remedy, but a system supporting comprehensive patient record integration remains elusive. Furthermore, the Office of the National Coordinator for Health Information Technology (ONC), in a 2015 Congressional report, detailed how technology vendors and providers limit patient access, by what has since been codified as information blocking [4,5]. Thus, not only do patient data remain disjointed but also barriers erected by data holders dissuade patient engagement and information exchange, culminating in the loss of agency. Blockchain technology coupled with nationally recognized interoperability standards (eg, Fast Healthcare Interoperability Resources, FHIR) has been presented as a viable solution to the said concerns [1-3,6-24].

Traditional health information exchange follows 1 of the following 3 models: push (sending information from 1 location to another), pull (extracting information from a source), or view (peering into a system). Although these practices technically achieve health information exchange, they are not sustainable, en masse solutions to patient-centered care. Thus, Halamka et al [23] proposed blockchains are a fourth model, with a stated goal of restoring patient agency $[3,24]$.

Blockchains are distributed and decentralized repositories of information secured by various cryptographic primitives. Ideally, participants (eg, patients, providers, and payers) upload data to the chain in a secure, authenticated fashion. The result is a comprehensive medical record accessible by those with patient permission as enforced by smart contracts. As participants only need to communicate with the blockchain using recognized interoperability standards (eg, FHIR), once trust is established, all information is securely exchanged. That is, instead of having multiple points of connection, document formats, and exchange protocols to follow (each a security risk and potentially costly to address), a universally accessible blockchain minimizes the overall risk to the participating entity while simultaneously enriching information exchange and patient engagement.

More specifically, deploying blockchain in health care is suggested to break down information exchange barriers inherent in disparate, siloed EHR systems; empower patients through data consolidation and access controls and enabling (eg, secure and verifiable authorizations, form completion, discharge instruction review, and patient-generated data contribution); improve quality of care while reducing costs and fraud; promote data integrity, validation, and provenance; track medical devices and pharmaceuticals; facilitate clinical trial accountability and auditability; and support research through access to large-scale, longitudinal, aggregated patient records [3,6-22]. Virtually, all previous works are proof of concepts or pilots, endeavoring to address the information security, interoperability, data integrity, identity validation, and scalability concerns hindering adoption [3,6-19].

Herein, we submit technical solutions to address these concerns, culminating in a detailed framework and open-source proof-of-concept tool-HealthChain - for a patient-centered blockchain. In the Methods section, HealthChain's components are defined and, when appropriate, compared with an immutable blockchain design. Contributions include a mixed-block blockchain, redactable patient blocks, amendable smart contracts, adoption of proxy re-encryption (PRE) for granting and revoking data sharing rights, formulation of a 2-party PRE decryption (2PD) scheme to facilitate mediated exchange, 4 configurable modes of operation, and a comprehensive set of experiments.

\section{Blockchain Applications in Health Care}

Heralded as a disruptive technology, blockchain research has intensified in recent years. Researchers and developers in health care have proposed, conceptualized, and implemented blockchain-based platforms to transform patient data sharing and information interoperability.

OmniPHR is a patient-centered blockchain emphasizing the distributed and interoperable principles of personal health records (PHRs). Patient data are recorded in encrypted, hierarchical blocks signed by the inserting entity (eg, provider, patient, or medical device). As data may be stored off-chain, OmniPHR maintains location pointers [25]. Another well-known system-MedRec-was conceived by researchers at Harvard and MIT. This Ethereum-based system links global patient identities to records held by providers. MedRec authenticates participants and stores provider pointers and record hashes (for data integrity). Patients interface with providers through MedRec to view data through smart contracts. Furthermore, patients manage third party access through the creation of smart contracts $[3,23,24]$. FHIRChain, developed by Zhang et al [2], is a blockchain-based architecture faceted in accordance with the secure and scalable sharing requirements of the ONC's Shared Nationwide Interoperability Roadmap [26] and leverages the emerging FHIR standard [27]. As with MedRec, data are stored off-chain and accessible through pointers and smart contract-controlled access tokens. In addition to interoperability, Kuo et al's [28] ModelChain performs privacy-preserving machine learning in the blockchain [28,29].

Beyond patient-centric applications, other blockchain solutions have been presented in a health care setting including supply chain management $[1,12,17,30-32]$, clinical research and data 
monetization [14,16,33-36], medical and research fraud detection [34,35,37], public health surveillance [13,18,20,38], and managing internet of (healthy) things [12,39-43].

\section{Background and Terminology}

\section{Consensus and Hashing}

A consensus algorithm is a protocol followed by the blockchain when determining the truthfulness and timeliness of blocks under consideration. On reaching consensus, blocks are accepted or rejected. There are many consensus algorithms from which to choose proof of work [44], proof of stake [45,46], proof of elapsed time [47,48], or Kafka [47,49]—a discussion of each is outside the scope of this work, and we refer the reader to the supplied references for further exploration.

Each block is identified by a hash, which is essentially a unique and verifiable fingerprint (Figure 1). A 1-way cryptographic hashing function produces said hash, representing the content of a message. In blockchain, the message consists of block data and the previous block's hash; the inclusion of the latter creates an unbreakable bond (ie, chain; Figure 2). Hashing functions satisfy 2 key principles: (1) each input has a distinct output (ie, uniqueness), and (2) a given input has the same output (ie, verifiability).

Point 1 seeks to prevent collisions - a phenomenon where 2 distinct messages have the same hash. Attackers can theoretically exploit collisions by forging blocks with desirable modifications (eg, a financial transfer), replacing authentic blocks as the forged hashes are verifiable (point 2) - this is an oversimplification but suitable for illustrative purposes. Thus, it is imperative to use hash functions without known vulnerabilities or collisions.

Figure 1. Block schematic with sample financial data and hashes.
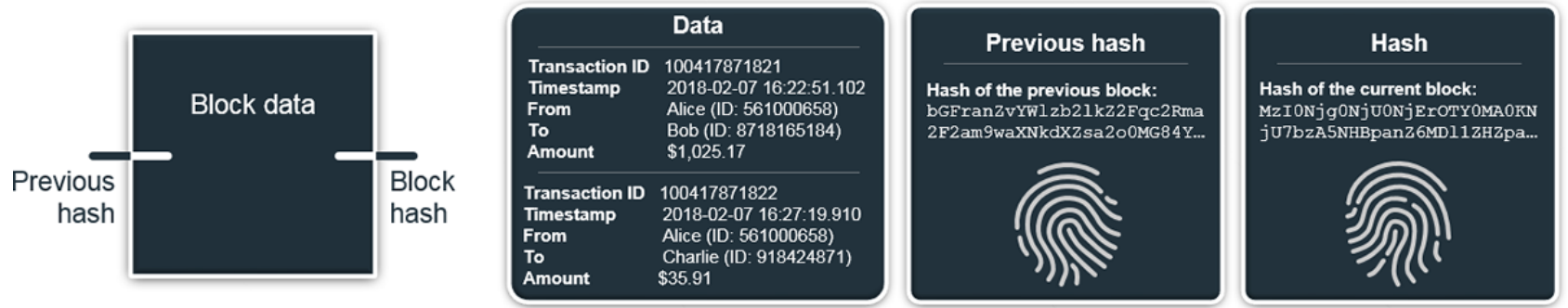

Figure 2. Blockchain diagram with several blocks, including the foundational genesis block, and noted hash connections.

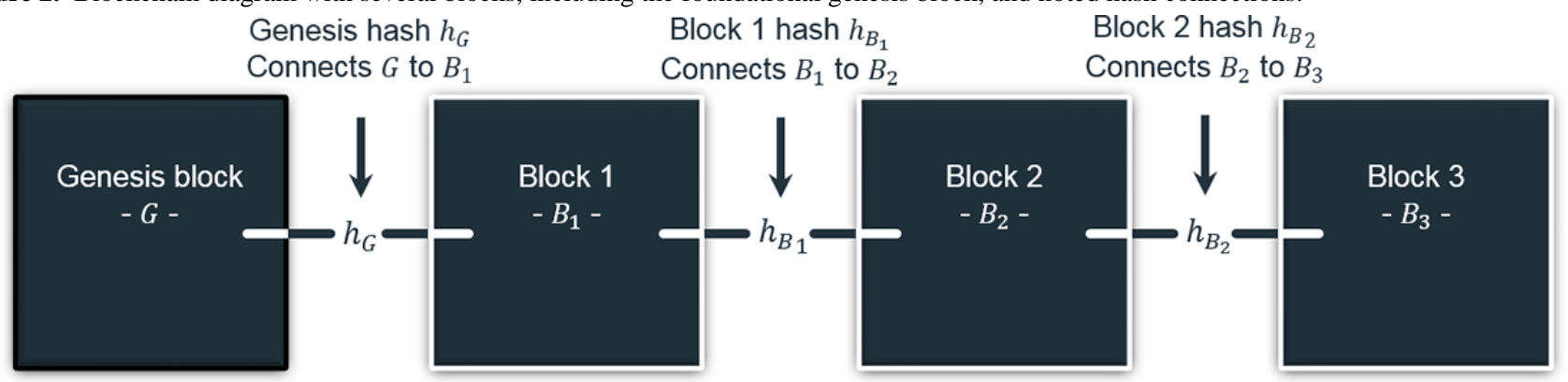

\section{Chameleon Hashing}

Before Ateniese et al's redactable blockchain [50], a block was axiomatically held as immutable. The authors, however, made several keen observations as to when mutability was desirous if not mandatory to comply with the legal, regulatory, and general usability requirements. These include legal violations (eg, illicit content or intellectual property rights infringements), amending changes to information (eg, avoiding data fragmentation [see section on Patient Blocks]), patching executable code (eg, debugging smart contracts or removing an embedded virus), expunging data (eg, right to be forgotten, General Data Protection Regulation, or privacy breach), and allowing for blockchain consolidation (eg, a merger) [7,8,15,50-52].

Chameleon hashing was posited as a viable alternative to traditional functions. As proposed by Krawczyk and Rabin [53-55], chameleon hashing satisfies the 2 hashing principles while introducing a trapdoor, allowing for efficient generation of collisions by the possessor of the trapdoor (ie, private) key.
However, without the said key, collisions are just as unlikely as nontrapdoor functions [50,53-55].

\section{Smart Contracts}

Smart contracts are autonomous transactions executed when stipulated terms of an agreement are met [3,22,51,52,56-59]. The creation of such contracts arose from the need to engender trust in an inherently untrustworthy environment. For instance, if some condition is met, how can one guarantee each party will comply with the agreement? Once executed, it will carry out the specified terms without fail.

Smart contracts, popularized by cryptocurrencies such as Bitcoin and Ethereum, have many practical applications in health care. Patients, for instance, can provide authorization through smart contracts to participate in studies or share information. They can codify rules leading to patient notification, for example, data accessed or communication received. They can also be used as a form of context-based access control, stipulating access rights to covered entities, business associates, and subcontractors $[3,21,22]$. 


\section{Proxy Re-Encryption}

PRE enables the delegation of decryption rights by a delegator to a delegatee through an intervening proxy [60-63]. The notion is quite intuitive and shown in Figure 3. A user, for example, Alice, encrypts a message forming a ciphertext. Bob requests a re-encryption key to facilitate decryption of Alice's ciphertext without exposing either party's secret information. PRE has been generally deployed in the cloud [63-70] for network storage [60], distributed file systems [71], email forwarding [65,71], and information exchange [72]. In health care, it has been suggested to safeguard patient data and identities in cloud-based systems $[73,74]$, secure mobile health monitoring and telehealth
[66-68,75], and control disclosure of information in PHRs [65] and health information exchanges [72].

Most PRE schemes use elliptic curve cryptography (ECC) [76-80], an asymmetric cryptosystem (Federal Information Processing Standard [FIPS] 186-4 [81]). Advancements in quantum computing [82-85] and modern attacks [86-88], however, foreshadow its demise. One promising replacement is quantum-resistant lattices [71,89-95]. Kirshanova [71] and Kim and Jeong [93] have recently published frameworks for implementing PRE using lattices. Thus, as we enter a postquantum age, so too will PRE, ensuring its longevity.

Figure 3. Proxy re-encryption process overview.
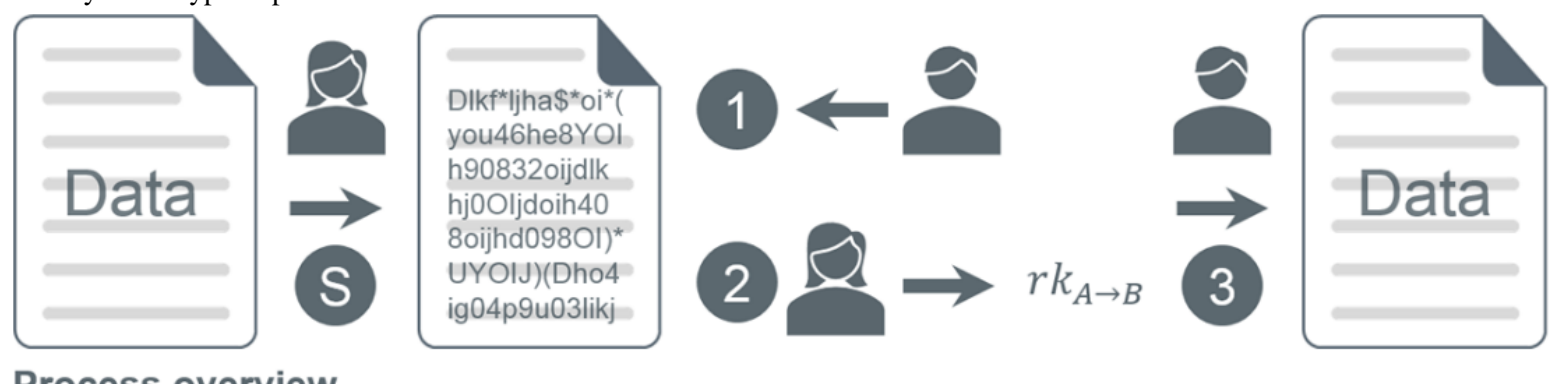

\section{Process overview}

Setup (S): Alice ( $A$, delegator) encrypts her data using proxy re-encryption and stores it.

1. Bob ( $B$, delegatee) requests access to Alice's data (directly (ie, here) or through a proxy).

2. Alice generates a re-encryption key $\left(r k_{A \rightarrow B}\right)$ using Bob's public key and her private key, sending the re-encryption key and encrypted data to Bob (or provides him with the data's location).

3. Bob uses a combination of his private key and the re-encryption key to decrypt Alice's data.

\section{Methods}

\section{Proposed HealthChain Framework}

Herein, an overview of the HealthChain framework is presented in Textbox 1. Specific details are provided in the proceeding subsections.

\section{Patient Centered}

As a patient-centric framework, HealthChain presents patients with a holistic view of their medical record, restoring agency through interaction. It encourages the accumulation, modification, generation, and review of information; ensures data integrity; authenticates identities; promotes unambiguous exchange; and executes user-granted access rights through smart contracts. Thus, HealthChain does not suffer from the common ailments plaguing PHR adoption such as data security and validity concerns, interoperability challenges, trust, and technological barriers to adoption [96-98]. Of note, HealthChain is not intended to replace EHRs but to serve as an interface between patients and third parties (eg, providers or payers).

\section{Permissioned Blockchain}

HealthChain is defined as a permissioned blockchain; only trusted parties (eg, hospitals, research institutions, universities, and government agencies) have the authority to manipulate the blockchain within a private network. These parties form a consortium (eg, a Regional HealthChain Organization) to manage the HealthChain, ensuring compliance with, for example, relevant statutes (eg, Health Insurance Portability and Accountability Act of 1996, HIPAA) and interoperability standards. Permissioning is further extended to patients, who are validated by a consortium member before account creation; this process can imitate those for patient portals. Although permissioning is a HealthChain requisite, a specific implementation is not. Thus, adopters may incorporate any system of choice.

Textbox 1. The 6 components of the HealthChain framework.

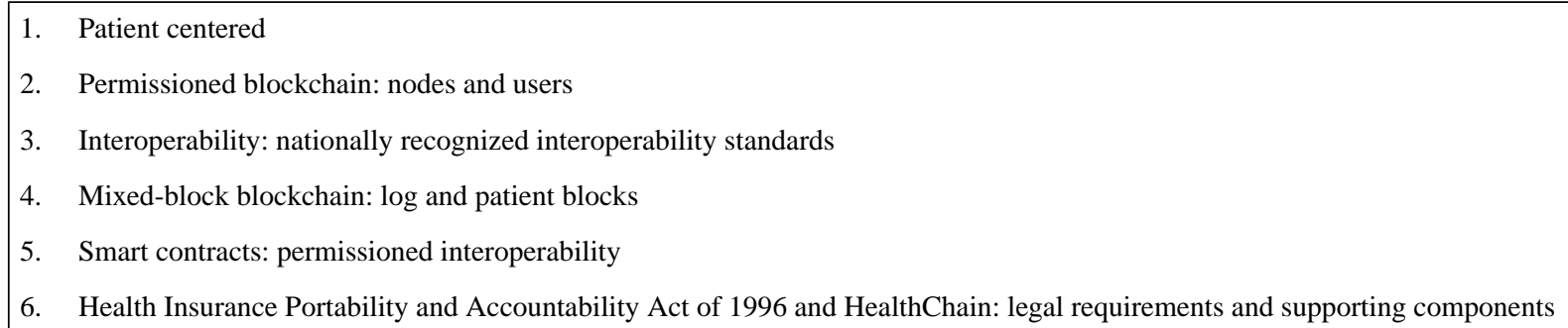




\section{Interoperability}

Patient-managed health information systems must conform to nationally recognized interoperability standards to be successful $[99,100]$. Using proprietary or lesser known standards (if at all) erects interoperability barriers, diminishing utility and adoption. Although any standard(s) is acceptable in this framework, HL7 FHIR [27] is recommended.

Although not yet federally required, HL7 FHIR is, according to the 2019 Interoperability Standards Advisory by ONC, under consideration for 26 interoperability needs [100]. Furthermore, ONC's Interoperability Proving Ground (an open community platform for sharing interoperability projects) indicates $21.3 \%$ (96/450) of projects use FHIR. Notable participants include
Allscripts, the American Medical Association, Cisco, NewYork-Presbyterian Hospital, OpenHIE, the Sequoia project, and the US Department of Veterans Affairs [101]. Apple has also invested in FHIR for their PHR app [102]. Clearly, the anticipation is standard acceptance, thus our selection.

\section{Mixed-Block Blockchain}

The proposed blockchain integrates 2 semantically distinct block types: $\log$ and patient, each detailed in subsequent sections. The distinction lies in block redaction or editability. Patient blocks are proposed to be redactable, whereas log blocks are not. Figure 4 augments Figure 2 to account for the 2 types. As detailed, the architecture is unaffected. A simple flag in each block distinguishes the types.

Figure 4. Mixed-block blockchain adaptation of Figure 2.

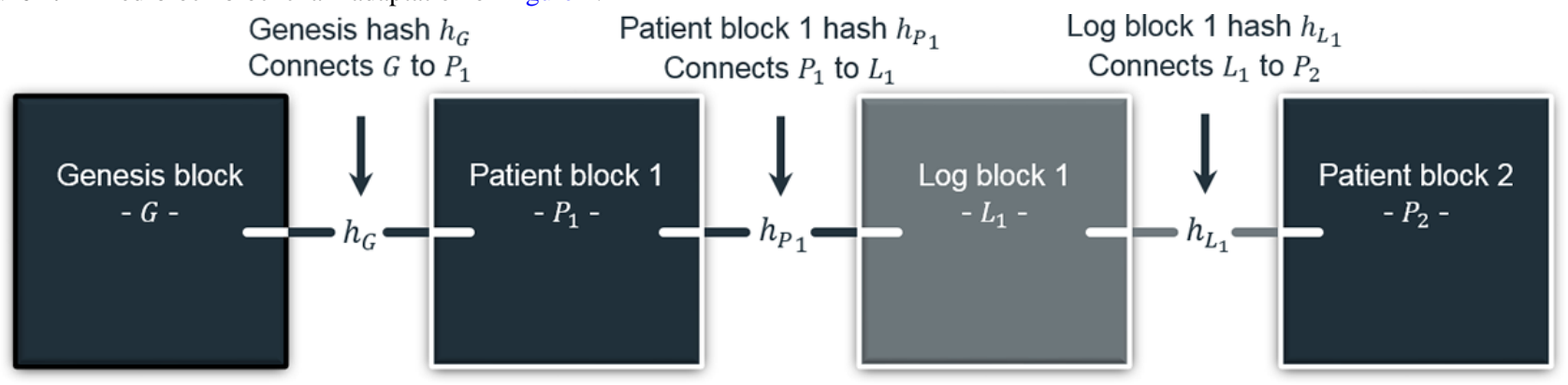

\section{Log Blocks}

Log blocks are an immutable, historical account of operations on the blockchain, such as added patients and blocks, patient block modification metadata, and the issuance and execution of smart contracts. Hence, traditional hashing algorithms (eg, Secure Hash Algorithm [SHA]-256) suffice. In addition, as the data are not sensitive (ie, contain no identifying information), encryption is unnecessary. Thus, log blocks are added to the blockchain following contemporary means (eg, through consensus).

\section{Patient Blocks}

Structurally, patient blocks consist of plaintext metadata (eg, unique identifier, type flag, patient's anonymous identifier, timestamp, hash, and the issuing miner's identifier and signature), encrypted patient data, and smart contracts. Principally, they adhere to Ateniese et al's redaction scheme [50] and are established and updated as follows.

When a patient requests an account, an authorized node prepares a block and submits it through a selected consensus method for inclusion. This represents the only instance of consensus in the patient block process (Figure 5, account flow). Once accepted, the patient assumes control. Block alterations are hashed, signed, and broadcasted to the network. Nodes validate the transactions and apply the addendums (Figure 5, redact flow). Multimedia data require special handling, as their large size disproportionately (likely prohibitively) consumes bandwidth and computational resources. We adopt the methodology in MedRec [3], where data remain at the source with a location reference stored in the block for ad-hoc retrieval. Throughout this process, transactions in the form of logs are collected. As stated in the previous section, log blocks are added following a traditional consensus methodology and the chain amended (Figure 5, log flow).

Redaction addresses 3 shortcomings of immutable blocks and patient transaction isolation. The first is data fragmentation. Immutable blockchains insert new transactions in contemporaneous blocks, splintering medical records, which are collections of temporal events, rather than isolated, for example, financial, transactions (Figure 6, initial and subsequent flow). Retrieval, consequently, necessitates (1) blockchain (or a potentially corruptible, off-chain index) scanning by nodes to recover and transmit encrypted fragments and (2) decrypting and reconstituting said fragments by requesters (Figure 7). The greater the fragmentation, the more resource intensive the process. Redaction, as implemented in HealthChain, colocates patient information (Figure 5, redact flow), minimizing overall system effort (Figure 7). Second is immutability itself. Legitimate modifications (eg, adding encounter notes, modifying medical histories, or removing incorrect user-generated data) are simulated in immutable blockchains through new transactions. Requesters must apply these transactions in the proper temporal order (eg, overwrite older data with newer) to reconstruct consistent records. Resources (ie, time, space, and bandwidth) are thus depleted with each modification. Redaction modifies data in place, nullifying this effect. The third, and final, shortcoming is consensus. Modifications produce new blocks necessitating consensus. Redactions to established blocks avoid this costly process, conserving time, effort, bandwidth, and storage for users and nodes. 
Figure 5. Patient account (ie, block) establishment, redaction, and logging processes in HealthChain.

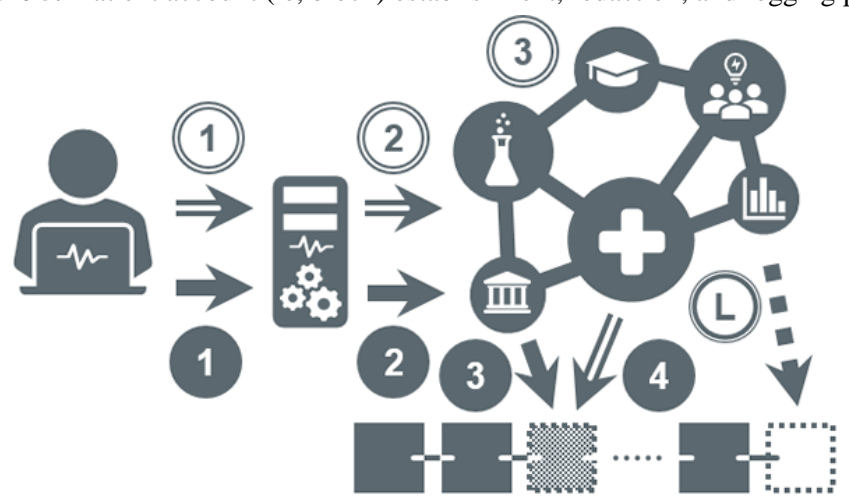

Account process overview

1. The patient connects to the HealthChain service and requests an account (ie, block).

2. The assigned node validates the patient, generates a block, and broadcasts it to the HealthChain network.

3. Consensus is immediately initiated.

4. If consensus is reached, the new patient block is appended to the blockchain.

L. A log transaction is generated, and traditional block construction and consensus is followed.

\section{Legend}

Process flow Data flow Blocks

$\begin{array}{lll}\text { (\#) Account } & \Rightarrow \text { Account } & \text { Existing } \\ \text { \# Redact } & \rightarrow \text { Redact } & \text { New } \\ \text { (ㄴ) Log } & \rightarrow \text { Log } & \text { Redacted }\end{array}$

\section{Redact process overview}

1. Some time later, the patient reconnects to the HealthChain service and submits a redaction, providing the signed and encrypted block.

2. The assigned node verifies the redacted block's hash and the patient's signature.

3. If valid, the node broadcasts the information over the HealthChain network and redacts its local instance (no consensus required).

L. A log transaction is generated, and traditional block construction and consensus is followed.

Figure 6. Initial and subsequent patient data entry in a traditional blockchain.
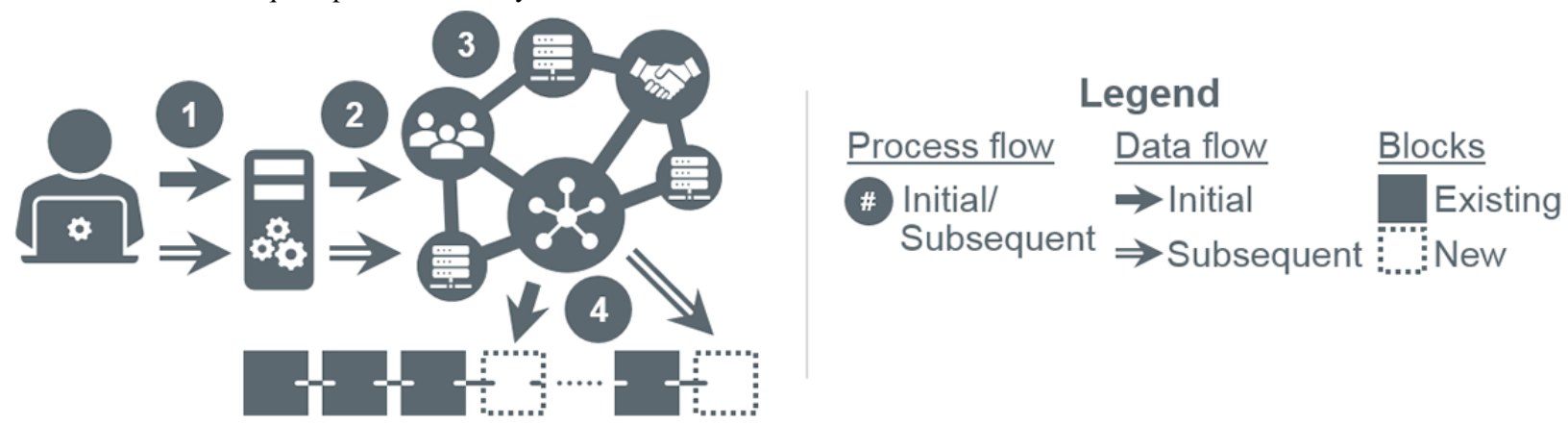

Initial and subsequent processes overview

1. The patient connects to a blockchain service and submits a transaction with new/updated information (time lapse depicted between the initial and subsequent transactions). Note: account generation is not portrayed as options abound, eg, from direct user action to organizationally assigned accounts.

2. The service broadcasts the transaction to nodes/miners/orders in the blockchain.

3. A block is constructed and consensus is initiated once specified conditions are met (eg, block size).

4. If consensus is reached, the block (encasing, in part, the new/updated data) is appended to the blockchain. 
Figure 7. Information retrieval in an immutable blockchain versus HealthChain.

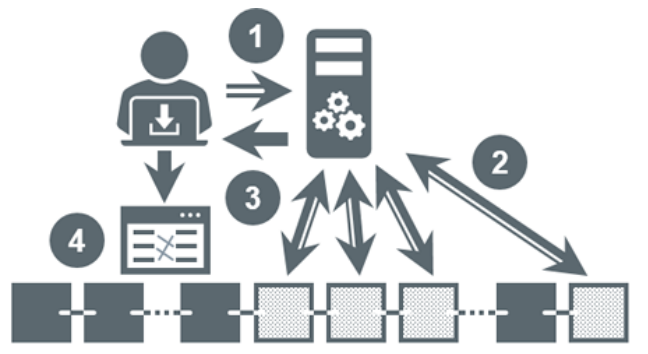

Immutable blockchain retrieval process

1. The patient connects to a blockchain service and submits a retrieval request.

2. The service, via a node (excluded for brevity), extracts the patient's encrypted transactions from various blocks (through an index or scan).

3. The record fragments are sent to the patient.

4. The patient decrypts and combines the data fragments to form a comprehensive record.

\section{Hashing Redactable Blocks}

Once appended to the blockchain, a block's hash cannot change; otherwise, the chain breaks. Redaction, therefore, precludes traditional hash functions (eg, SHA-256) as, by definition, different inputs produce distinct hashes. Hence, the deployment of chameleon hashing.

Ateniese et al [50] identified several chameleon hash formulations suitable for blockchains. Here, public-coin chameleon hashing $(\mathrm{PCCH})$ is applied. $\mathrm{PCCH}$ uses public-key cryptography for verification (using public keys) and redaction (by generating collisions with private keys). In terms of security, $\mathrm{PCCH}$ hashes are as hard to forge as nontrapdoor functions. Hence, $\mathrm{PCCH}$ maintains a secure and valid state in redactable blockchains [50].

\section{Block Encryption}

Classified as electronic protected health information under the HIPAA, patient data must be encrypted (45 Code of Federal Regulations [CFR] section 164.304) per the US Department of Health and Human Services-issued guidelines (Health Information Technology for Economic and Clinical Health 13402(h)(2)). These guidelines reference National Institute of Standards and Technology Special Publication 800-111, which recommends the Advanced Encryption Standard (AES), although any FIPS-approved cryptosystem (eg, ECC) is acceptable [103]. Any selection must be mindful of the proposed framework, which includes information exchange.

Traditional symmetric (eg, AES) and asymmetric (eg, ECC) primitives are insufficient as they compel 1 of the 3 insecure or infeasible information exchange solutions [69]. The first is exchanging secret information. This jeopardizes data integrity (through altering, corrupting, or re-encrypting data) and digital identities (secured by private keys). Second, originators (eg, patients) can re-encrypt data under requester public keys. Although secure, originators must be omnipresent and dedicate considerable personal resources to the process; otherwise, sharing ceases. Finally, third parties can represent originators for re-encryption. However, this exposes plaintext and secret information to the said third parties. As a potential solution to these challenges, we endorse PRE.

PRE facilitates information exchange through dedicated third parties without exposing sensitive information. Beyond proposing PRE, we identified 4 operations critical to securing and validating stored and exchanged data in the proposed framework: (1) encryption and decryption, (2) re-encryption, (3) sign and verify (eg, digital signatures), and (4) encrypt sign and decrypt-verify (eg, encrypted sign and verify). AFGH (so named for the authors' last names) [60] is the chosen PRE scheme as it fulfills operations 1 and 2 and encourages the formulation of 3 and 4 . In addition, its re-encryption keys are unidirectional (decrypt only), noninteractive (no secret information exchanged), and nontransitive (cannot combine keys to forge privileges) [60]; Multimedia Appendix 1 provides a more thorough introduction.

Operations 1 and 2 are instrumental in securing and sharing sensitive information and are the foundation of any PRE scheme. Operation 3 facilitates message authentication and data integrity. A message signed (ie, encrypted) by a sender using its private key can be verified (ie, decrypted) by anyone with its public key. As only the sender has its private key, verification proves authenticity and integrity. Multimedia Appendix 1 offers our posited sign and verify AFGH modification. Operation 4 unites 1 and 3 to protect sensitive, signed messages. Signed messages are encrypted with the recipient's public key, ensuring only it can decrypt before verification. Multimedia Appendix 1 presents the conceived encrypt-sign and decrypt-verify AFGH extension.

Moreover, some PRE-derived ciphertexts (eg, ElGamal based [104] in AFGH) are malleable. This is advantageous when optimizing, for instance, data rekeying, which amounts to multiplication in ElGamal, instead of decrypt-encrypt in AES. In addition, it is compulsory for homomorphic encryption, that is, computation on ciphertexts. Although this is an active area of research, fully homomorphic schemes (ie, those that add and multiply) are presently impractical [94,95,105-111]. The research, however, is progressing, with lattices emerging as a promising area $[94,95,105,106]$. Lattices, therefore, have the 
potential to bring postquantum, fully homomorphic encryption to this framework.

PRE is traditionally deployed for key exchange $[60,76]$. Data are encrypted using, for example, AES, and the key encrypted and exchanged through PRE. An alternative, as proposed herein, is PRE-encrypted data. Both have merits and were extensively tested. Table 1 compares these approaches by 4 properties. We focus on PRE implementations over elliptic curves (ECs) because of their prominence [76-80].

EC PRE is slower and larger than AES because of key and bilinear map sizes. EC keys are twice AES for the same cryptographic strength-for example, EC-256 equates to AES-128 [112]. Bilinear maps (see Multimedia Appendix 1) are even larger-for example, up to 3072 bits for EC-256 [112-115]. Consequently, the system uses more space and time, hindering performance. Furthermore, AFGH eliminates re-encryption data integrity concerns through decrypt-only keys [60], a property unsupported by AES. Finally, the malleable AFGH cipher supports dynamic rekeying (ie, altering encryption keys) through multiplication, instead of the delegator having to decrypt, encrypt, and retransmit all data as under AES.

Table 1. A comparison of Advanced Encryption Standard encrypted blocks with proxy re-encryption encrypted keys to proxy re-encryption encrypted blocks.

\begin{tabular}{lll}
\hline Property & AES block encryption & PRE $^{\mathrm{b}}$ block encryption \\
\hline Encrypt and decrypt speed & Faster & Slower \\
Size of ciphertext & Smaller & Larger \\
Key operations & Decrypt and encrypt & Decrypt only \\
Rekeying ciphers & Decrypt then encrypt & Multiplication \\
\hline
\end{tabular}

${ }^{\mathrm{a}} \mathrm{AES}$ : Advanced Encryption Standard.

${ }^{b}$ PRE: proxy re-encryption.

\section{Smart Contracts}

Smart contracts herein enable conditional information exchange. Their mutability (from patient block storage) permits modification and revocation without duplicate, conflicting, or vulnerable contracts remaining on the blockchain. During instantiation, a template is automatically populated (once authorization is furnished); no programming is necessary. On execution, an engine hardcoded into the server platform applies a series of instructions, given a contract's parameters. There are several reasons for this approach. The proposed smart contracts are structurally uniform (eg, identifiers and signatures of involved parties, terms, and re-encryption keys), eliminating the need for arbitrary code support. Furthermore, logical errors [56-58] and exploitable vulnerabilities [51,52,58,59] in programmed contracts can compromise patient data. An automated, templatized design with a parameterized, hardcoded engine eliminates these vulnerabilities.

Figure 8 demonstrates the smart contract initiation process, in which a re-encryption key is created and stored in a signed contract on the delegator's block. Figure 9 shows the execution of the contract generated in step 1 by the delegatee in step 2, facilitating data decryption. In this example (and proof of concept), data are stored as FHIR messages for direct interoperability with capable systems. This represents a basic application of PRE to smart contracts. It is, however, insecure.

Although granting access with PRE is simple, revoking it is not. Consider the following, 2 parties enter into a 1-week smart contract. If the delegatee notes the re-encryption key during valid execution, nothing explicitly prevents it from decrypting the delegator's block after contract termination.

A naïve refinement to close this vulnerability is implementing PRE as originally defined - proxies decrypt delegator data, then encrypt for delegatees [61]. However, as numerous studies have concluded, proxies cannot be trusted with delegator private keys and plaintext $[65,70,90,116]$. Another approach is subkeying by, for instance, time [60,117-119]. Each period has a unique, random variable to which all ciphertexts and re-encryption keys are bound. It is argued that this ensures delegatees cannot access new information. However, if a contract is terminated within a period, new information will be available as access is not revoked, only confined. In addition, one must manage many keys, data are fragmented over time, and interperiod interoperability is cumbersome.

To address revocation, we submit 2PD (Multimedia Appendix 1), a variant of the original formulation [61] whereby data decryption requires 2 parties with complementary re-encryption keys. Figures 10 and 11 adapt Figures 8 and 9, respectively, to 2PD. In premise, the intermediary (eg, node; an augmented proxy, thus the distinction) applies its re-encryption key to the delegator's data (as it is malleable), producing a ciphertext discernable to the delegatee alone. In terms of security, intermediaries no longer require private keys, and its re-encryption key does not expose plaintext. Furthermore, delegatee re-encryption keys cannot decrypt data on the blockchain, thus realizing revocation. 
Figure 8. Smart contract initiation using standard proxy re-encryption.

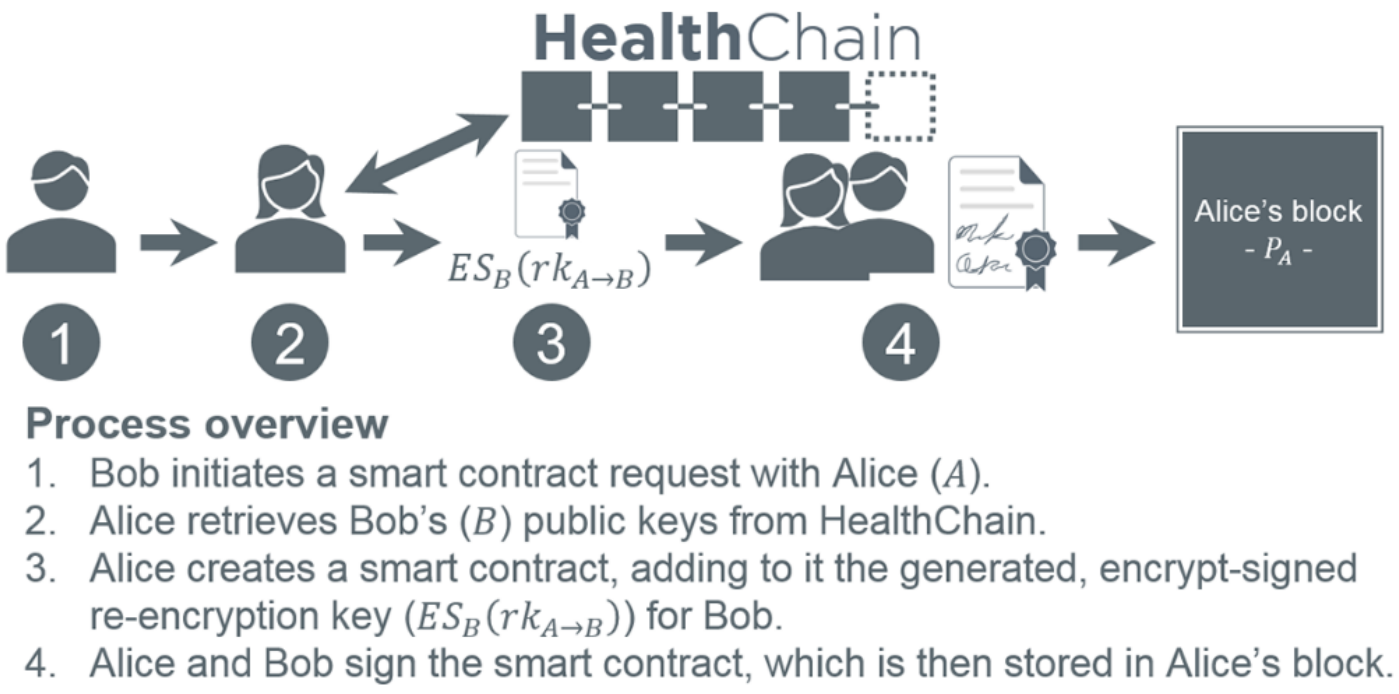

Figure 9. Smart contract execution using standard proxy re-encryption. FHIR: Fast Healthcare Interoperability Resources.
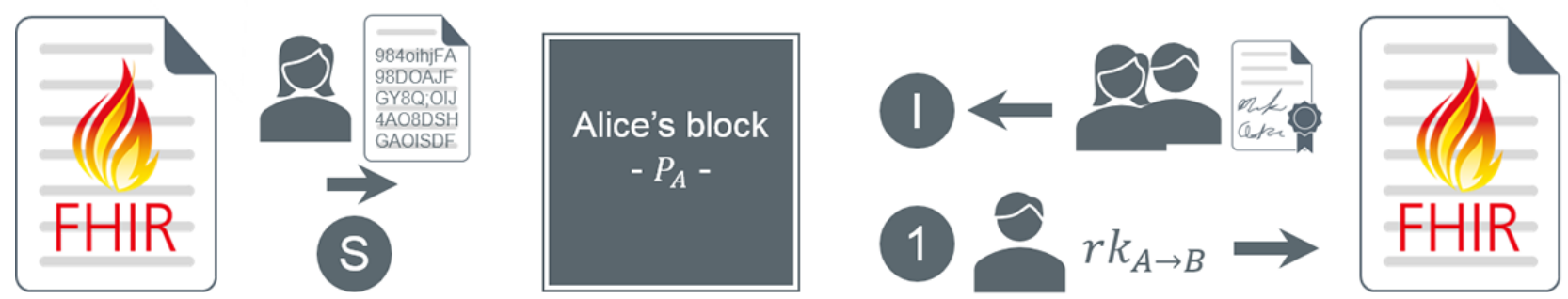

\section{Process overview}

Setup (S): Alice $(A)$ encrypts her information and stores the results in her block. Initialize (I): Alice and Bob $(B)$ enter into a smart contract, with the results stored in Alice's block.

1. Bob executes the smart contract in Alice's block, retrieves the re-encryption key $\left(E S_{B}\left(r k_{A \rightarrow B}\right)\right)$, decrypt-verifies it (obtaining $r k_{A \rightarrow B}$ ), then combines it with his private key to decrypt Alice's block.

Figure 10. Smart contract initiation using 2-party proxy re-encryption decryption.

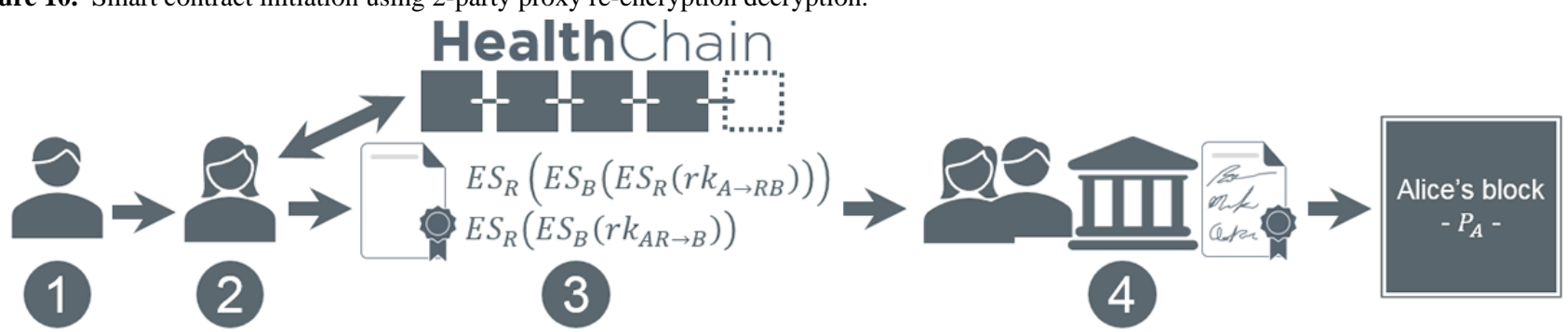

\section{Process overview}

1. Bob $(B)$ initiates a smart contract request with Alice $(A)$ and intermediary $(R)$.

2. Alice retrieves Bob's and the intermediary's public keys from HealthChain.

3. Alice creates a smart contract, adding to it the generated, layered, encrypt-signed re-encryption keys for Bob and the intermediary $\left(E S_{R}\left(E S_{B}\left(r k_{A \rightarrow R B}\right)\right)\right.$ and $E S_{R}\left(E S_{B}\left(E S_{R}\left(r k_{A R \rightarrow B}\right)\right)\right)$ respectively).

4. Alice, Bob, and the intermediary sign the smart contract, which is then stored in Alice's block. 
Figure 11. Smart contract execution using 2-party proxy re-encryption decryption. FHIR: Fast Healthcare Interoperability Resources; PRE: proxy re-encryption.

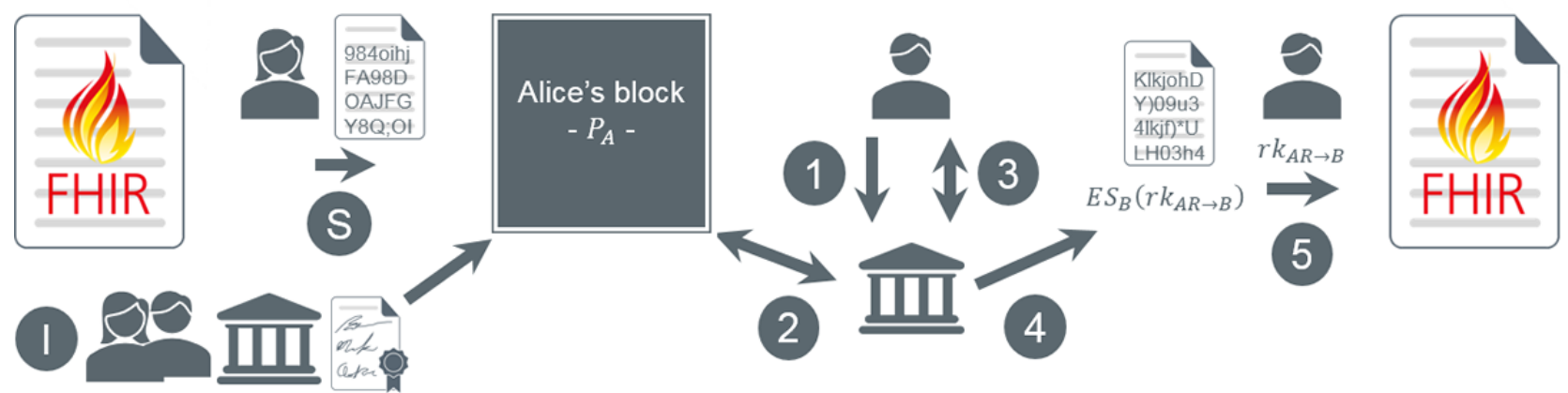

\section{Process overview}

Setup $(\mathrm{S})$ : Alice $(A)$ encrypts her information and stores the results in her block.

Initialize (I): Alice, Bob $(B)$, and intermediary $(R)$ enter into a smart contract, with the results stored in Alice's block.

1. Bob executes the smart contract via the intermediary.

2. The intermediary validates the smart contract via Alice's block, and, if valid, retrieves the necessary information.

3. The intermediary begins the decrypt-verify process on the re-encryption keys with Bob, resulting in the intermediary re-encryption key $\left(r k_{A \rightarrow R B}\right)$ and an encrypt-signed key for Bob $\left(E S_{B}\left(r k_{A R \rightarrow B}\right)\right)$.

4. The intermediary re-encrypts Alice's PRE-encrypted information with $r k_{A \rightarrow R B}$ (no plaintext access), sending it and the encrypt-singed re-encryption key $E S_{B}\left(r k_{A R \rightarrow B}\right)$ to Bob.

5. Bob uses a combination of his private key and the decrypt-verified re-encryption key $r k_{A R \rightarrow B}$ to decrypt the data sent by the intermediary (cannot directly decrypt Alice's information).

There are several additional 2PD factors to consider. First, it demands an intermediary be semitrusted in that it will not maliciously alter the hardcoded smart contract engine or distribute keys to unauthorized entities. As our framework implements a permissioned blockchain, intermediaries (ie, nodes) are inherently trustworthy. That said, private keys and plaintext are withheld to deter collusion and improper data use. Second, re-encryption keys must mathematically prohibit manipulation resulting in forged privileges. This is imparted by AFGH's nontransitive property [60]. Finally, re-encryption key retrieval mandates intermediary and delegatee consent without revealing said keys. Encrypt-sign layering, as documented in Figures 10 and 11, provides such support, as 1 layer must be decrypt verified by the opposing entity (ie, consent) and the final layer by the intended recipient, simultaneously averting unilateral access and key exposure.

\section{Health Insurance Portability and Accountability Act of 1996 and HealthChain}

Administrative rule 45 CFR section 164.524 grants patients the right to request copies of their records, which are to be delivered in the form and format requested by the individual, if they are readily producible in such as format (45 CFR section 164.524(c)(2)(i)). With the growing acceptance of HL7 FHIR (section Interoperability), one can envisage a future with it being readily producible. Thus, the proposed framework can leverage patient access rights through FHIR to seamlessly communicate with health information systems, eliminating the burden of ad-hoc data extraction, manual data entry, and data transformation placed on users and providers.

Systems in this space must be HIPAA compliant. Table 2 maps facets of the posited framework to pertinent HIPAA administrative rules, suggesting compliance. A thorough assessment, however, must be conducted before deployment. 
Table 2. Health Insurance Portability and Accountability Act of 1996 administrative rule specifications (privacy rule and security rule) and submitted HealthChain components supporting compliance.

\begin{tabular}{|c|c|c|}
\hline Specification & Rule: 45 CFR $^{\mathrm{a}}$ section 164 & HealthChain \\
\hline Authorization and revocation $\left(\mathrm{PR}^{\mathrm{b}}\right)$ & 508,510 & $\begin{array}{l}\text { Smart contracts, sign and verify, and encrypt-sign and decrypt-verify: } \\
\text { confidential communications and verifiable requests and authorizations }\end{array}$ \\
\hline Restriction requests (PR) & $522(a)(1)$ & $\begin{array}{l}\text { Smart contracts, sign and verify, and encrypt-sign and decrypt-verify: } \\
\text { confidential communications and verifiable requests and authorizations }\end{array}$ \\
\hline Amendments (PR) & 526 & $\begin{array}{l}\text { Smart contracts, sign and verify, and encrypt-sign and decrypt-verify: } \\
\text { confidential communications and verifiable requests and authorizations }\end{array}$ \\
\hline Confidential communications (PR) & $522(b)(2)$ & $\begin{array}{l}\text { Encrypt-sign and decrypt-verify: message integrity, verifiable identity, } \\
\text { and encryption }\end{array}$ \\
\hline Unique user authentication $\left(\mathrm{SR}^{\mathrm{C}}\right)$ & $312(a)(2)(i)$ & $\begin{array}{l}\text { Unique encryption and hashing key pairs, sign and verify, and encrypt- } \\
\text { sign and decrypt-verify: verifiable identity (key possession and signing) } \\
\text { and patient block hashes and patient block encryption }\end{array}$ \\
\hline Encryption and decryption (SR) & 312(a)(2)(iv) & $\begin{array}{l}\text { Unique encryption and hashing key pairs, sign and verify, and encrypt- } \\
\text { sign and decrypt-verify: verifiable identity (key possession and signing) } \\
\text { and patient block hashes and patient block encryption }\end{array}$ \\
\hline Integrity (SR) & $312(\mathrm{c})(1)$ & $\begin{array}{l}\text { Unique encryption and hashing key pairs, sign and verify, and encrypt- } \\
\text { sign and decrypt-verify: verifiable identity (key possession and signing) } \\
\text { and patient block hashes and patient block encryption }\end{array}$ \\
\hline Audit controls (SR) & 312(b) & Log blocks \\
\hline Person or entity authentication (SR) & $312(d)$ & $\begin{array}{l}\text { Sign and verify, encrypt-sign and decrypt-verify, re-encryption key layer- } \\
\text { ing, and delegatee re-encryption: verifiable identity (verification algorithms } \\
\text { and construction of the delegatee re-encryption process) }\end{array}$ \\
\hline $\begin{array}{l}\text { Transmission security-integrity controls } \\
\text { and encryption (SR) }\end{array}$ & 312(e)(1), (2)(i), and (2)(ii) & $\begin{array}{l}\text { Patient block encryption, intermediary re-encryption, sign and verify, and } \\
\text { encrypt-sign and decrypt-verify (layering): verifiable identity and transfer } \\
\text { of encrypted data only by design }\end{array}$ \\
\hline
\end{tabular}

${ }^{\mathrm{a} C F R}$ : 45: Code of Federal Regulations.

${ }^{\mathrm{b}} \mathrm{PR}$ : privacy rule.

${ }^{\mathrm{c}} \mathrm{SR}$ : security rule.

\section{Experimental Design}

The experimental design facilitates the examination of HealthChain's novel components. Additional services such as permissioning and consensus along with a comprehensive distributed network were not evaluated for the following reasons. First, although essential to the framework's practical implementation (whereas here we are exploring new functionality), no improvements to those areas were proposed in this work; hence, testing is unwarranted. Second, ancillary services and an arbitrarily sized experimental network inject considerable overhead (potentially orders of magnitude above the measured item) into the process, rending the subject of analysis indistinguishable from noise. As such, we intentionally limited the components implemented in the proof of concept to only those necessary to successfully evaluate the processes defined in the subsequent sections.

Regarding experimental block operations, recall that as patient blocks are generated during account initialization, all subsequent actions necessitate redactions to said block section Patient Blocks). Therefore, all experiments conducted herein are redactions.

\section{Configurable Modes}

HealthChain operations are dictated by 4 configurable modes (see Multimedia Appendix 2 for a detailed summary). The first

is the 2-option block encryption mode: (1) AES-encrypted data with PRE-encrypted key (denoted as A) and (2) PRE-encrypted data (signified by P), as defined and justified in section Block Encryption.

The second is the storage mode with 2 possibilities: full block (F) and incremental (I). Full block incorporates all data into a single block. Incremental, in all but 1 case (see next mode), transmits only new and modified entries or removal instructions. Table 3 compares the 2 options over 6 properties. Full block transmits 1 block (ie, transaction), which is more efficient than multiple blocks. Cipher padding in full block mode is negligible, whereas potentially considerable in incremental mode (cumulative padding). Record isolation is trivial in incremental mode as each is its own entry. Full block masks transactions in an encrypted block, making isolation and metadata attacks difficult. Regarding transmission speed and size, incremental mode may be smaller and thus faster if only transmitting minimal changes relative to the medical record. Finally, a single block is bound in size by the storage mechanism (eg, a database byte array attribute). Larger medical records may require multiple blocks, fragmenting the information and increasing the complexity of management. Incremental does not suffer from this limitation. 
Table 3. Comparison of full block and incremental storage mode options.

\begin{tabular}{lll}
\hline Property & Full block & Incremental \\
\hline Transactions & 1 & 1 or more \\
Cipher padding & Negligible & Potentially considerable \\
Record isolation & No & Yes \\
Speed of transmission & Slow & Potentially fast \\
Size of transmission & Large & Potentially small \\
\hline
\end{tabular}

Third is encryption key mode, with static (S) and dynamic (D) choices. Static mode encrypts a patient block using the same key in perpetuity, whereas dynamic mode re-encrypts data under a new one with each update. Dynamic mode enhances security, as compromised information is of limited use, but consumes more resources. AES-encrypted data in incremental block storage (AI) mode requires the patient to re-encrypt and transmit all data. Being malleable, PRE-encrypted data in incremental storage (PI) mode encrypts updates using a new key, sending them and a scalar to a node for appending and rekeying, respectively.
Server-side encryption mode is the last mode: simply on $(\mathrm{Y})$ or off $(\mathrm{N})$. If enabled, the server encrypts (by block encryption mode) user data using an ephemeral key for each entry, renewed under the dynamic key policy (does not impact PRE operations). This protects against improper access by dynamically rekeying accessed entries.

Tables 4 and 5 present listings of configurable mode abbreviations and descriptions used in our experiments by AES and PRE encryption respectively. Refer to Multimedia Appendix 2 for more details.

Table 4. Advanced Encryption Standard (AES) configurable experimental mode abbreviations and descriptions.

\begin{tabular}{|c|c|}
\hline Mode & Description \\
\hline $\mathrm{AF}$ & AES-encrypted data, full block storage \\
\hline AI & AES-encrypted data, incremental storage \\
\hline $\mathrm{ADF}$ & AES-encrypted data, dynamic encryption key full block storage \\
\hline ADI & AES-encrypted data, dynamic encryption key incremental storage \\
\hline ASF & AES-encrypted data, static encryption key full block storage \\
\hline ASI & AES-encrypted data, static encryption key incremental storage \\
\hline AFN & AES-encrypted data, full block storage no server-side encryption \\
\hline AFY & AES-encrypted data, full block storage server-side encryption \\
\hline AIN & AES-encrypted data, incremental storage no server-side encryption \\
\hline AIY & AES-encrypted data, incremental storage server-side encryption \\
\hline ADFN & AES-encrypted data, dynamic encryption key, full block storage, no server-side encryption \\
\hline ADFY & AES-encrypted data, dynamic encryption key, full block storage, server-side encryption \\
\hline ADIN & AES-encrypted data, dynamic encryption key, incremental storage, no server-side encryption \\
\hline ADIY & AES-encrypted data, dynamic encryption key, incremental storage, server-side encryption \\
\hline ASFN & AES-encrypted data, static encryption key, full block storage, no server-side encryption \\
\hline ASFY & AES-encrypted data, static encryption key, full block storage, server-side encryption \\
\hline ASIN & AES-encrypted data, static encryption key, incremental storage, no server-side encryption \\
\hline ASIY & AES-encrypted data, static encryption key, incremental storage, server-side encryption \\
\hline
\end{tabular}


Table 5. Proxy re-encryption (PRE) configurable experimental mode abbreviations and descriptions.

\begin{tabular}{|c|c|}
\hline Mode & Description \\
\hline $\mathrm{PF}$ & PRE-encrypted data, full block storage \\
\hline PI & PRE-encrypted data, incremental storage \\
\hline PDF & PRE-encrypted data, dynamic encryption key full block storage \\
\hline PDI & PRE-encrypted data dynamic encryption key incremental storage \\
\hline PSF & PRE-encrypted data, static encryption key full block storage \\
\hline PSI & PRE-encrypted data, static encryption key incremental storage \\
\hline PFN & PRE-encrypted data, full block storage no server-side encryption \\
\hline PFY & PRE-encrypted data, full block storage server-side encryption \\
\hline PIN & PRE-encrypted data, incremental storage no server-side encryption \\
\hline PIY & PRE-encrypted data, incremental storage server-side encryption \\
\hline PDFN & PRE-encrypted data, dynamic encryption key, full block storage, no server-side encryption \\
\hline PDFY & PRE-encrypted data, dynamic encryption key, full block storage, server-side encryption \\
\hline PDIN & PRE-encrypted data, dynamic encryption key, incremental storage, no server-side encryption \\
\hline PDIY & PRE-encrypted data, dynamic encryption key, incremental storage, server-side encryption \\
\hline PSFN & PRE-encrypted data, static encryption key, full block storage, no server-side encryption \\
\hline PSFY & PRE-encrypted data, static encryption key, full block storage, server-side encryption \\
\hline PSIN & PRE-encrypted data, static encryption key, incremental storage, no server-side encryption \\
\hline PSIY & PRE-encrypted data, static encryption key, incremental storage, server-side encryption \\
\hline
\end{tabular}

\section{Experiments}

A total of 5 system dimensions are measured over 16 mode combinations for AES-128 and EC-256: transmission size, network latency, client processing time, server processing time, and smart contract execution.

Transmission size refers to the number of bytes sent from client to server. Typical consumer internet connections have low upload rates as households consume more content than contribute; thus, upload bandwidth is a concern. Scalability for users with metered connection is also of interest, as they may incur costs associated with overages or plan adjustments.

Network latency assesses the effect internet-based transmissions have on the proposed framework. The results are analyzed independently and integrated into client processing time and smart contract execution.

Client processing time represents the time devoted by clients to, for example, insert records, generate synchronization instructions, regenerate smart contracts (if dynamic keying), compute block hashes, and broadcast the previous to the blockchain.

Server processing time is the time incurred by servers during, for example, AES PRE key renewing (dynamic AES), instruction application (all), PRE scalar multiplication (PDIN/Y), and smart contract updating (all dynamic).

Smart contract execution measures the time required to run a smart contract. The process uses $2 \mathrm{PD}$ and writes the output to a zipped file on the delegatee's machine.

\section{Datasets}

Each of the 16 mode combinations was evaluated by insertion and scaling costs. Insertion costs are determined by adding records en masse to a clean system instance (only contains the account request profile). Insights are garnered on cost amortization and limits associated with bulk and single record processing without existing record influences (eg, re-encryption and retransmission), which may direct policy on block synchronization. Moreover, 4 datasets of observations (1 per day) were synthetically generated for testing (Table 6). Each was statically sized at 400 bytes for record-level evaluation. As each record is of identical size, cryptographic processing time, cipher length, and bytes transmitted are comparable by record across the various configurations.

Scaling is scrutinized by gauging the effect existing records have on insertions. As this system accumulates records, these experiments facilitate the examination of existing medical records on overall performance. For insertion, care was taken to avoid interaction between new and existing data. Here, the reverse is of interest on how existing data affect record insertions. The results inform decisions on configuration selection and synchronization strategy. Testing began by instantiating a system with 1 of the 3 datasets (Table 6) - representative of small, medium, and large patient records from a deidentified instructional medical database [120]. Then, the 4 insertion datasets were applied, with the average per record reported as an indicator of general performance. 
Table 6. Number of records and byte range per record by experimental dataset (all records were formatted as Health Level-7 Fast Healthcare Interoperability Resources [FHIR] JSON messages using HAPI FHIR).

\begin{tabular}{llll}
\hline Experiment & Records & Bytes/record (average) & Notes \\
\hline Insertion & 1 & 400 & 1 day \\
Insertion & 30 & 400 & 30 days \\
Insertion & 365 & 400 & 1 year \\
Insertion & 1461 & 400 & 4 years \\
Scaling & 334 & $395-761(581)$ & 26 encounters, 33 conditions, 145 medication requests, and 130 observations \\
Scaling & 945 & $395-768(675)$ & 27 encounters, 159 conditions, 624 medication requests, and 135 observations \\
Scaling & 2361 & $394-770(732)$ & 109 encounters, 119 conditions, 2029 medication requests, and 104 observations \\
\hline
\end{tabular}

\section{Testing Environment}

The testing environment aligns with the minimal requirements defined in the Experimental Design section. In its simplest form, HealthChain is a medium of information exchange between an entity (eg, patient) and a server (eg, node). Every process in HealthChain can be reduced to a series of entity-server interactions; therefore, our testing environment emulates this 2-machine structure.

The first machine is a Lenovo T540p running Windows 7 Enterprise with 16 GB of memory, an Intel i7-4800 MQ processor, and a wired, consumer internet connection. The second is a Dell Optiplex 9010 running Linux Mint 17.1 with 8 GB of memory, an Intel i7-3770 processor, and a wired, business internet connection. Communication rates (download/upload in megabits per second [Mbps]) are as follows: $32.1 / 5.9$ and 955.4/176.3 Mbps for each respective machine [121].

To facilitate direct processing time comparisons between client and server, 1 machine (the Lenovo) assumed both roles. This colocation, however, failed to address networking concerns. Thus, experiments incorporating transmission costs (ie, client processing time and smart contract execution) were duplicated using both machines, which are situated 5 miles apart. These times replaced those in the 2 identified measures for a more realistic outcome while still affording client and server relative performance comparisons.

\section{Proof-of-Concept Implementation}

The proof of concept facilitates the examination of the novel HealthChain elements as specified in section Experimental Design; it is not a production-ready blockchain system. The realization of the proposed framework requires the blending of the components defined herein with an existing blockchain technology such as Hyperledger Fabric or Ethereum. The proof of concept consists of 2 systems and 2 libraries written primarily in Java 8 and JSP and uses HAPI FHIR for document formatting [122] (Multimedia Appendix 3).

The first system, HealthChainServer, instantiates a single node that, for instance, establishes the blockchain, processes patient account requests, validates and manages patient identities and block transactions, supports 2PD, and transmits updates to patients (eg, the latest encounter). A multinode system (and therefore broadcasting capabilities) is unnecessary for testing.
In addition, log blocks are not examined as they are a trivial extension to existing blockchain technologies.

The second system, HealthChainWebClient, is a simple JSP-enabled version of the Gentelella Alela template [123]. Through the Web portal, patients can, for example, request accounts, create and manage smart contracts, manually add records, import and export FHIR messages, and receive updates (eg, from a participating hospital). It was through the file upload feature that experimental data were added, which were then transmitted to the server over a socket connection. Although sufficient for testing, a robust, security-focused, application programming interface-driven approach (such as the one developed using SMART on FHIR [124]) should be implemented before use in production.

Regarding libraries, the first provides chameleon hash support by way of PCCH [50] as outlined in the section on Hashing Redactable Blocks. The second realizes PRE through AFGH [60] (using, as a foundation, the Java Pairing-Based Cryptography library [jPBC] [113]) as justified in the section on Block Encryption.

\section{Results}

\section{Transmission Size}

Here, transmission size is analyzed by insertion (Figure 12) and scaling (Figure 13). Server-side encryption does not impact transmission, hence its exclusion.

In Figure 12, PDI and PSI are nearly double the others (in overall and per record transmission size). Both have $789-400=389$ bytes of padding (ie, wasted space) per record inserted, thus the disparity with all AES configurations. In addition, PDF and PSF are in line with AES (about 2\% larger) rather than PDI and PSI. This is attributable to the full block generation process, as all records are fused into one, then divided into 789-byte ciphers, markedly reducing padding (see the section on Dataset Effects on Cipher Size). Furthermore, by 365 , all ciphers near saturation (ie, negligible padding and amortized overhead).

Regarding overhead, dynamic options include 9137 bytes per smart contract (one here), ADI and ADF incorporate a new 397-byte AES PRE key, PDI uses a 384-byte scalar, and all send a 96-byte block hash. 
To scaling (Figure 13), ASI, PDI, and PSI are unaffected by the number of existing records (only transmit modifications), requiring 416,789 , and 810 bytes on average of encrypted data per added record, respectively.

Figure 12. Transmission size in kilobytes and bytes per record by the number of records inserted. ADF: AES-encrypted data, dynamic keys, full block storage; ADI: AES-encrypted data, dynamic keys, incremental storage; AES: Advanced Encryption Standard; ASF: AES-encrypted data, static keys, full block storage; ASI: AES-encrypted data, static keys, incremental storage; PDF: PRE-encrypted data, dynamic keys, full block storage; PDI: PRE-encrypted data, dynamic keys, incremental storage; PRE: proxy re-encryption; PSF: PRE-encrypted data, static keys, full block storage; PSI: PRE-encrypted data, static keys, incremental storage.

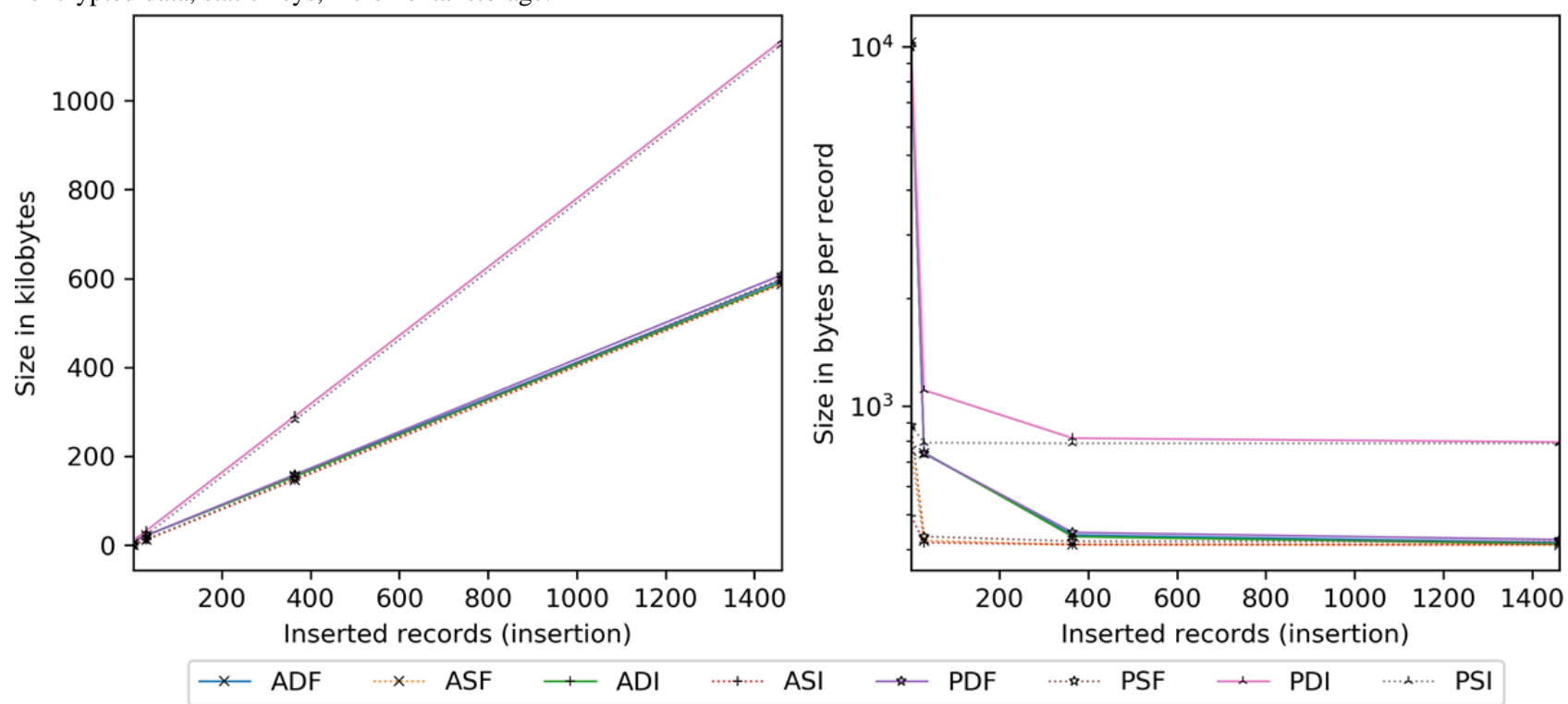

Figure 13. Transmission size in kilobytes per record added given an existing record set. ADF: AES-encrypted data, dynamic keys, full block storage; ADI: AES-encrypted data, dynamic keys, incremental storage; AES: Advanced Encryption Standard; ASF: AES-encrypted data, static keys, full block storage; ASI: AES-encrypted data, static keys, incremental storage; PDF: PRE-encrypted data, dynamic keys, full block storage; PDI: PRE-encrypted data, dynamic keys, incremental storage; PRE: proxy re-encryption; PSF: PRE-encrypted data, static keys, full block storage; PSI: PRE-encrypted data, static keys, incremental storage.

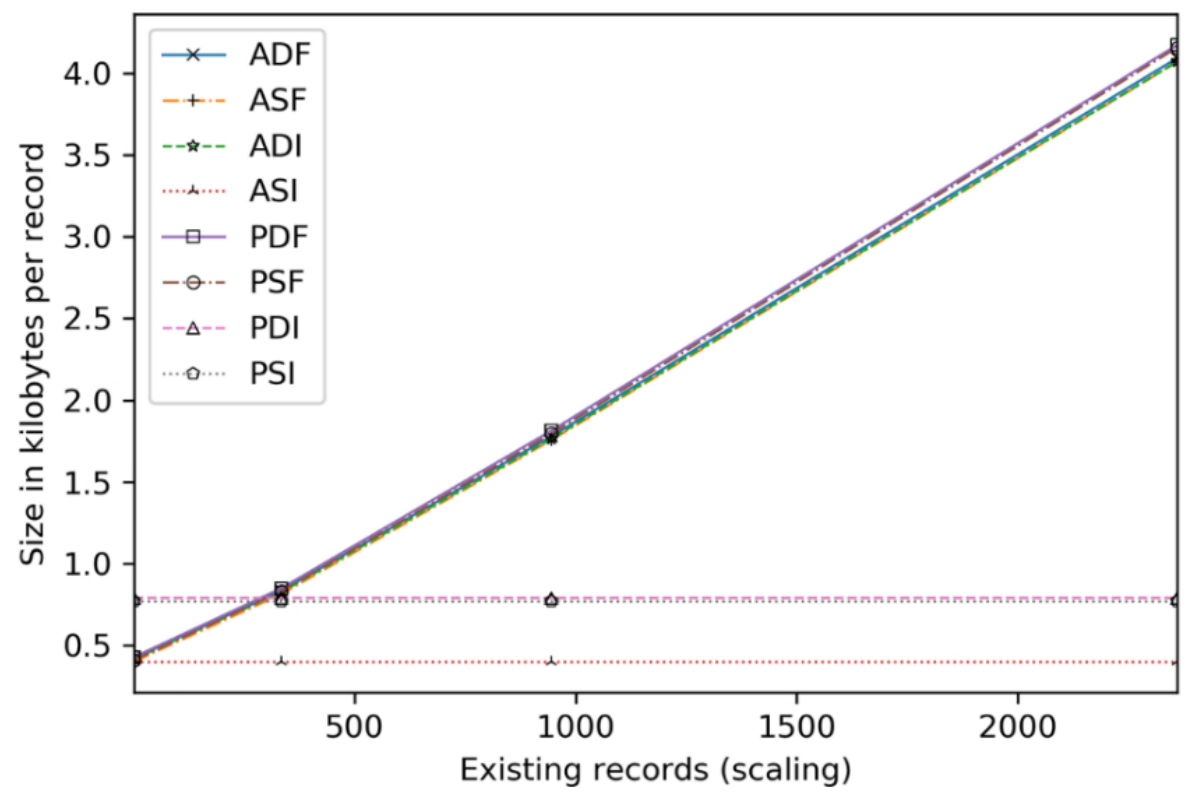

\section{Dataset Effects on Cipher Size}

AES and PRE cipher sizes are dissected in Tables 7 and 8 for incremental and full block encryption modes, respectively. Comparisons are drawn at the record level; thus, overhead bytes were removed (see the section on Transmission Size and 231 bytes for the patient record generated during account activation).
AES is slightly larger on average than the underlying data $(0.7 \%-4.1 \%)$ regardless of mode. PRE incremental is extensively padded (26.5\%-97.3\%), whereas minimal in full block mode (3.1\%-5.2\% beyond 1 insertion). Thus, PRE is subject to extreme variability, relative to AES, on input file size. 
Table 7. Incremental storage: byte range per file and average Advanced Encryption Standard- and proxy re-encryption-encrypted cipher sizes by dataset (insertion and scaling).

\begin{tabular}{llll}
\hline Dataset & Bytes/file range (average) & AES $^{\text {a }}$ average (difference, \%) & PRE $^{\mathrm{b}}$ average (difference, \%) \\
\hline 130,365, and 1461 & 400 & $416(4.0)$ & $789(97.3)$ \\
334 & $395-761(581)$ & $585(0.6)$ & $802(38.0)$ \\
945 & $395-768(675)$ & $679(0.7)$ & $840(24.3)$ \\
2361 & $394-770(732)$ & $737(0.7)$ & $926(26.5)$ \\
\hline
\end{tabular}

${ }^{\mathrm{a} A E S}$ : Advanced Encryption Standard.

${ }^{b}$ PRE: proxy re-encryption.

Table 8. Full block storage: total bytes per dataset and average Advanced Encryption Standard- and proxy re-encryption-encrypted cipher sizes by dataset (insertion and scaling).

\begin{tabular}{llll}
\hline Dataset & Total bytes & AES average (difference, \%) & ${\text { PRE }{ }^{\mathrm{b}} \text { average (difference, \%) }}^{789(97.3)}$ \\
\hline 1 & 400 & $416(4.0)$ & $12,624(5.2)$ \\
30 & 12,000 & $12,368(3.1)$ & $153,066(4.8)$ \\
365 & 146,000 & $150,112(2.8)$ & $613,053(4.9)$ \\
1465 & 584,400 & $600,848(2.8)$ & $200,406(3.4)$ \\
334 & 193,815 & $196,304(1.3)$ & $658,026(3.1)$ \\
945 & 637,934 & $644,688(1.1)$ & $1,779,981(3.0)$ \\
2361 & $1,727,714$ & $1,744,032(0.9)$ & \\
\hline
\end{tabular}

${ }^{a}$ AES: Advanced Encryption Standard.

${ }^{b}$ PRE: proxy re-encryption.

\section{Network Latency}

Network latency is analyzed in isolation to understand client-to-server (Figures 14 and 15) and server-to-client (Figure 16) effects. For insertion costs, Figure 14, incremental PRE grows at about twice the pace of others, proportional to cipher size (Tables 7 and 8). In addition, by 365 in Figure 15, the Mbps transmitted saturate the connection, whereupon ADI/ASI and PDI/PSI stabilize at 0.6 and 1.1 milliseconds per fragment (ie, a single record or full block), respectively. Regarding scaling (Figure 14), ASI, PDI, and PSI are constant, whereas full block and ADI grow as they reprocess all entries per update.
Figure 16 examines server-to-client transmissions as anticipated during record downloads from participating entities (eg, clinics) and intermediate smart contract results (if a delegatee). Data include the insertion sets as well as existing with 1461 additions to demonstrate scale. Transmission time depends on the block encryption (cipher size) and storage (padding effects) modes. $\mathrm{AF}, \mathrm{AI}$, and $\mathrm{PF}$ are indistinguishable from one another, whereas PI is on average 55\% slower because of larger, excessively padded ciphers. Regarding bandwidth, a limit at approximately 18.4 Mbps (3.4 times the update limit in Figure 15), first experienced by the larger PI, is revealed. This corresponds to increased trajectories in time. 
Figure 14. Client-to-server network latency in seconds per inserted record (ie, insertion) and seconds per record added given an existing record set (ie, scaling) - includes connection establishment, termination, and transmission time. ADF: AES-encrypted data, dynamic keys, full block storage; ADI: AES-encrypted data, dynamic keys, incremental storage; AES: Advanced Encryption Standard; ASF: AES-encrypted data, static keys, full block storage; ASI: AES-encrypted data, static keys, incremental storage; PDF: PRE-encrypted data, dynamic keys, full block storage; PDI: PRE-encrypted data, dynamic keys, incremental storage; PRE: proxy re-encryption; PSF: PRE-encrypted data, static keys, full block storage; PSI: PRE-encrypted data, static keys, incremental storage.
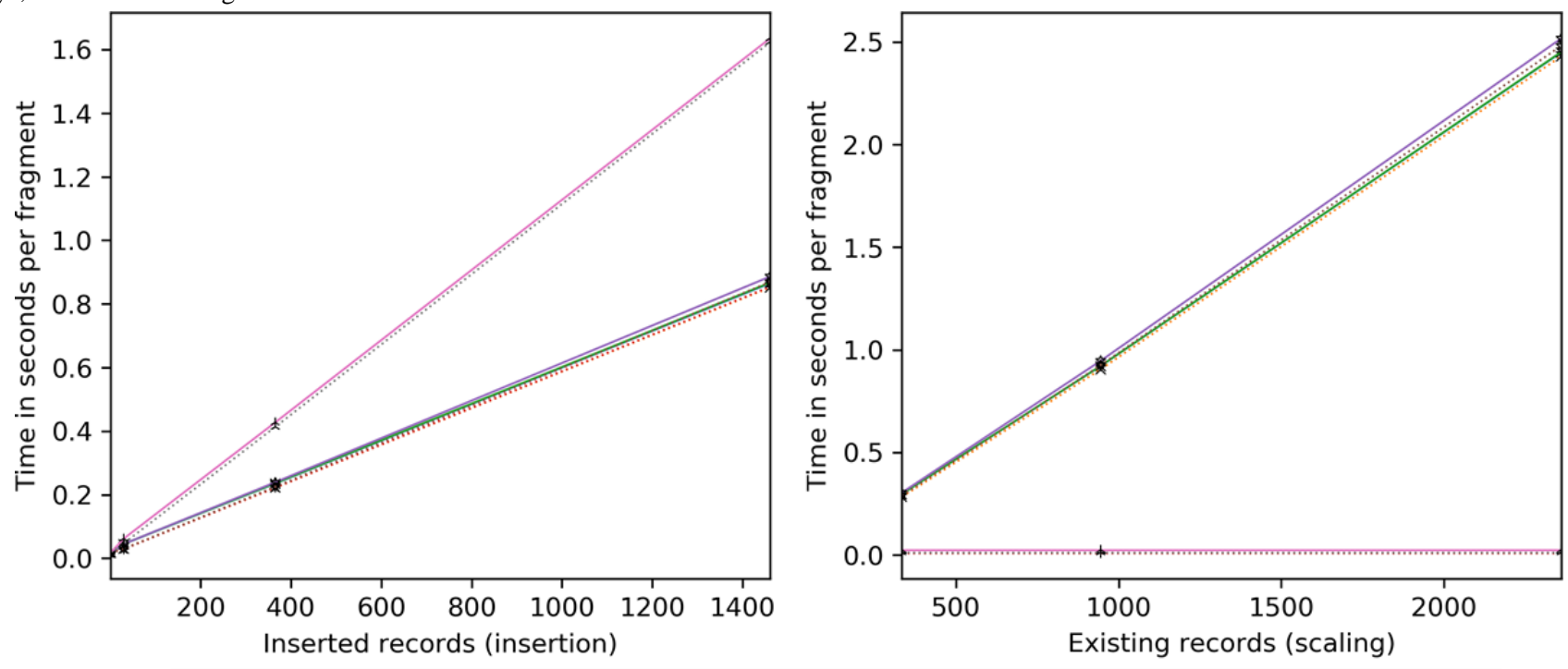

$\rightarrow$ ADF $\cdots \cdots$ ASF $\quad+$ ADI $\cdots \cdots+\cdots$ ASI

PDF

$\therefore$ PDI

PSI

Figure 15. Client-to-server network latency (transmission only) measured in megabits per second and milliseconds per fragment by the number of records inserted. ADF: AES-encrypted data, dynamic keys, full block storage; ADI: AES-encrypted data, dynamic keys, incremental storage; AES: Advanced Encryption Standard; ASF: AES-encrypted data, static keys, full block storage; ASI: AES-encrypted data, static keys, incremental storage; PDF: PRE-encrypted data, dynamic keys, full block storage; PDI: PRE-encrypted data, dynamic keys, incremental storage; PRE: proxy re-encryption; PSF: PRE-encrypted data, static keys, full block storage; PSI: PRE-encrypted data, static keys, incremental storage.
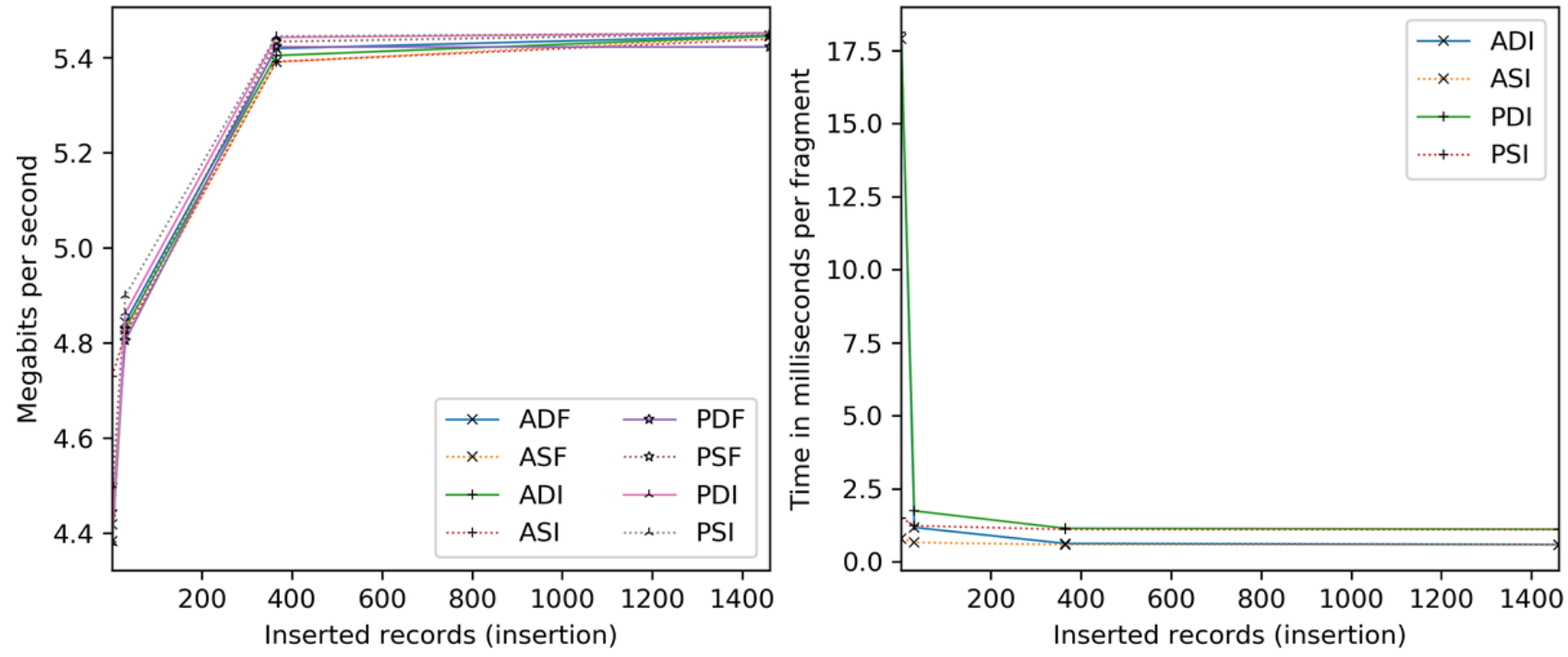
Figure 16. Server-to-client network latency (transmission only) measured in milliseconds and megabits per second by the number of records transmitted to the client. AES: Advanced Encryption Standard; AF: AES-encrypted data, full block storage; AI: AES-encrypted data, incremental block storage; PF: PRE-encrypted data, full block storage; PI: PRE-encrypted data, incremental storage; PRE: proxy re-encryption.

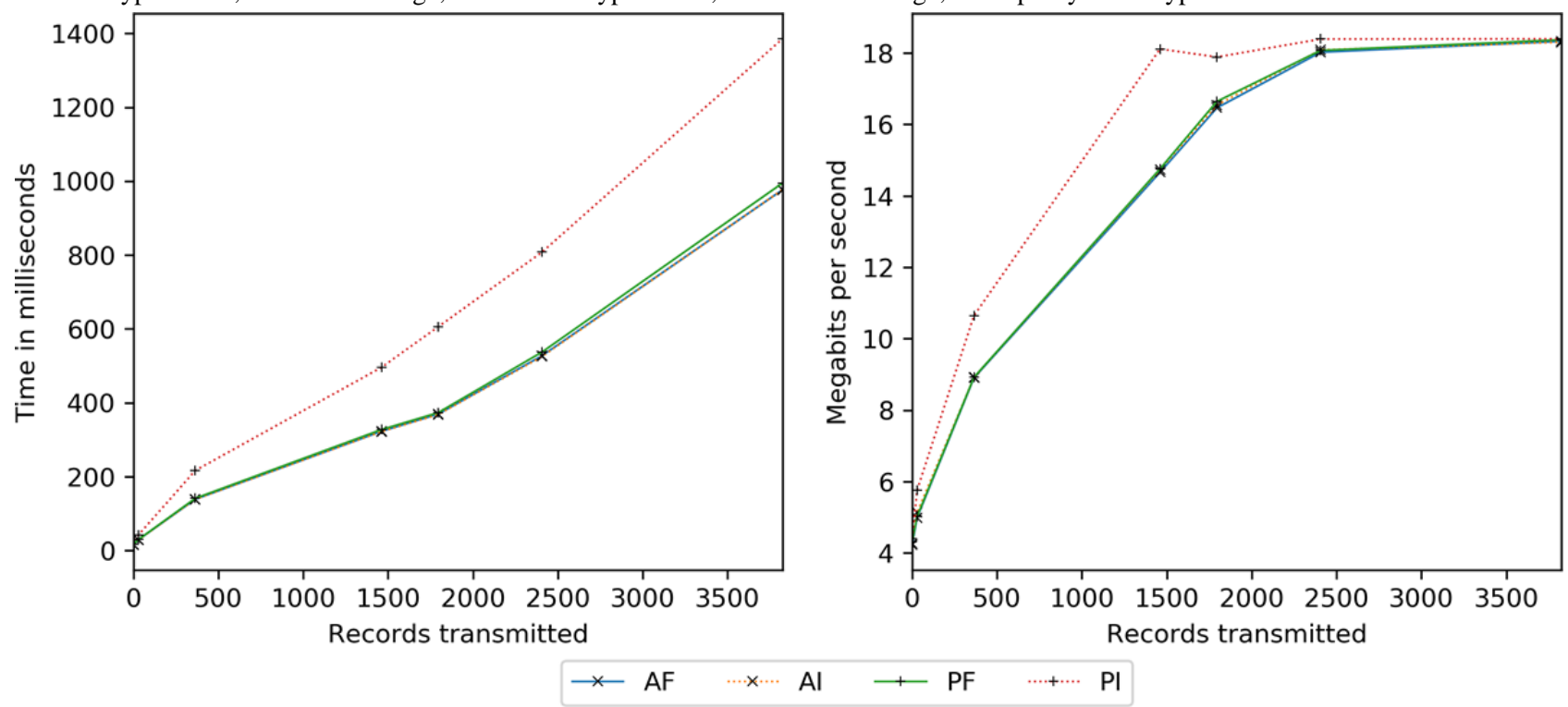

\section{Client Processing Time}

Client processing time is explored for insertions (Figure 17) and scaling (Figure 18). Server-side encryption does not impact client performance, hence its exclusion. For these tests, the client and server are the same machine (for relative comparison), whereas the transmission time is taken from the network latency experiments.

All configurations require a similar amount of time per Figure 17. The quickest are the static full block approaches, followed by dynamic full block ( $2 \%$ slower), static incremental $(8 \%$ slower), and dynamic incremental (12\% slower) approaches. Per record, by 365 , all are within 5 milliseconds and narrowing.

Concerning scaling (Figure 18), network latency accounts for $24 \%$ to $27 \%$ of the overall cost at 1 existing record, dropping precipitously to $1 \%$ to $4 \%$ by 365 . Beyond 365 , ASI, PDI, and PSI are constant time as only new and modified information are processed. Although the others are 4 to 9 milliseconds apart, ASF and PSF tend to be slightly faster. However, by 2362, ASI, PDI, and PSI are $68 \%$ to $71 \%$ more efficient than the other methods.

Figure 17. Client processing time in seconds and milliseconds per record by the number of records inserted. ADF: AES-encrypted data, dynamic keys, full block storage; ADI: AES-encrypted data, dynamic keys, incremental storage; AES: Advanced Encryption Standard; ASF: AES-encrypted data, static keys, full block storage; ASI: AES-encrypted data, static keys, incremental storage; PDF: PRE-encrypted data, dynamic keys, full block storage; PDI: PRE-encrypted data, dynamic keys, incremental storage; PRE: proxy re-encryption; PSF: PRE-encrypted data, static keys, full block storage; PSI: PRE-encrypted data, static keys, incremental storage.
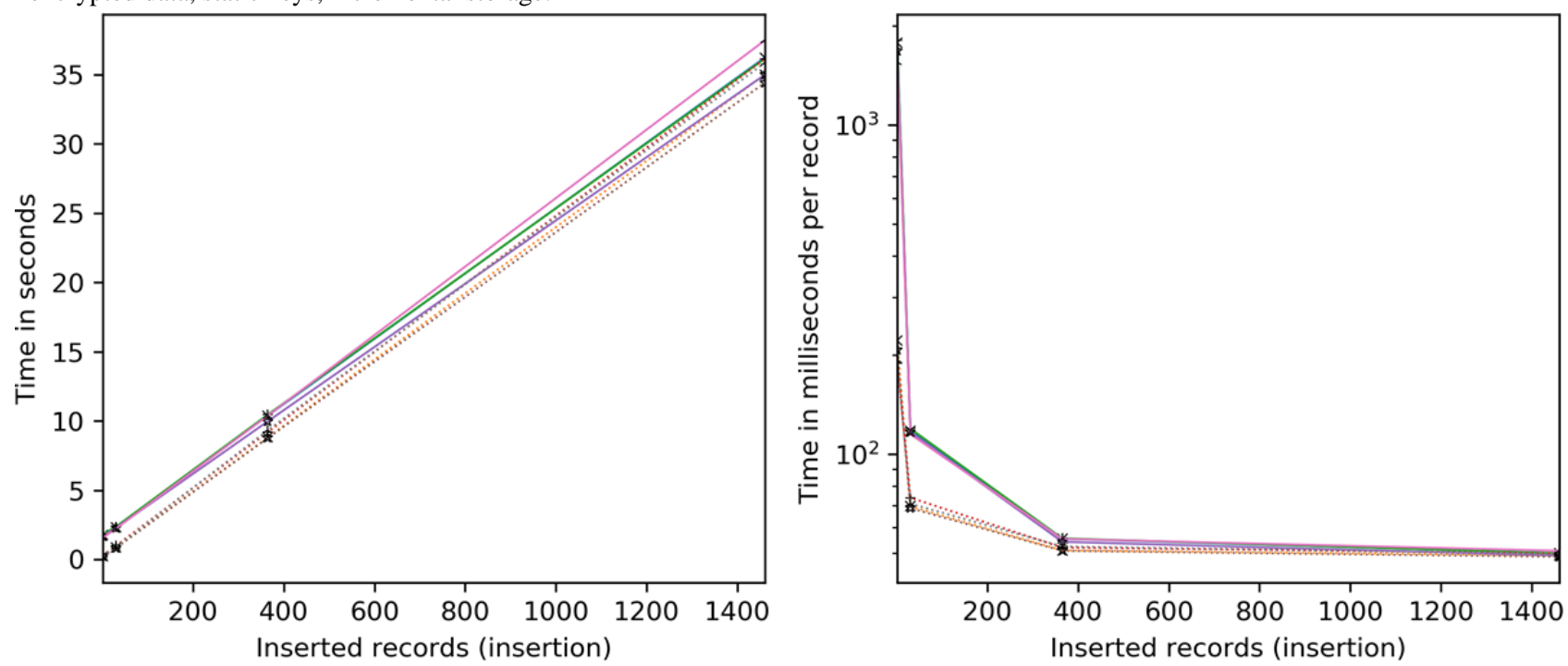

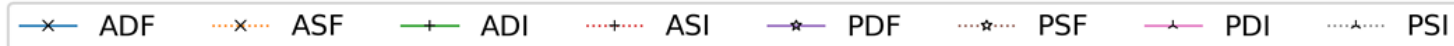


Figure 18. Client processing time in milliseconds per record added given an existing record set. ADF: AES-encrypted data, dynamic keys, full block storage; ADI: AES-encrypted data, dynamic keys, incremental storage; AES: Advanced Encryption Standard; ASF: AES-encrypted data, static keys, full block storage; ASI: AES-encrypted data, static keys, incremental storage; PDF: PRE-encrypted data, dynamic keys, full block storage; PDI: PRE-encrypted data, dynamic keys, incremental storage; PRE: proxy re-encryption; PSF: PRE-encrypted data, static keys, full block storage; PSI: PRE-encrypted data, static keys, incremental storage.

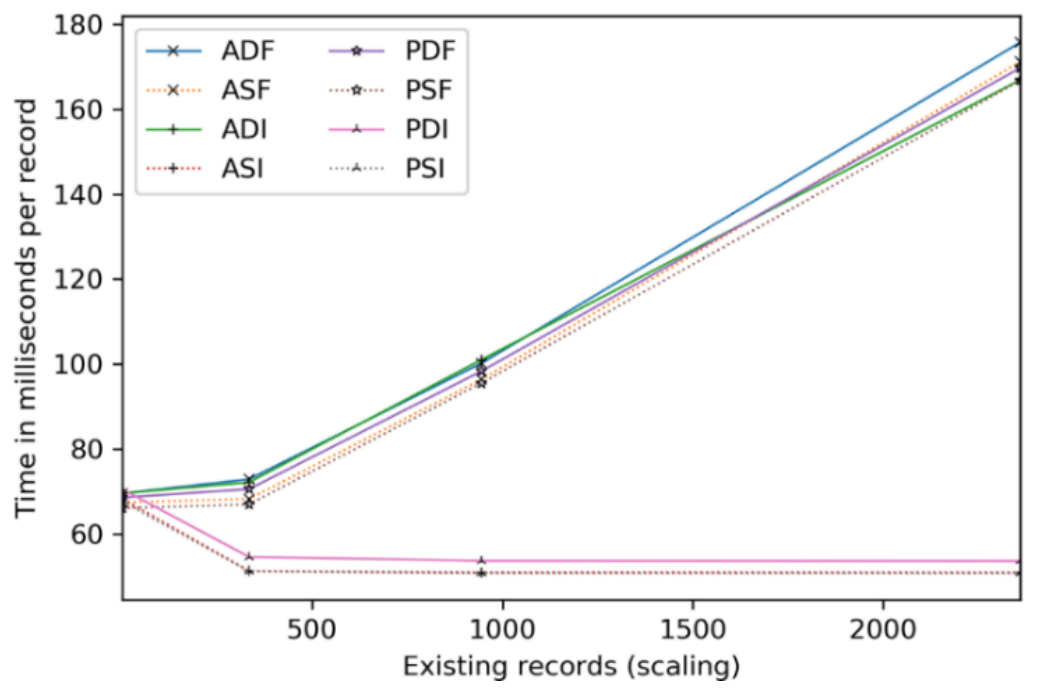

\section{Server Processing Time}

Server insertion costs are presented in Figure 19. Full block approaches process insertions the fastest. The order is in tens of milliseconds for AES (19-65 milliseconds), and PRE without server-side encryption (14-53 milliseconds). PRE with server-side encryption is measured in the 111 to 199 milliseconds range. ASIN and PSIN extend nearly uniformly from 7 to 889 milliseconds over the sets, with a slight dynamic keying penalty of 12 to 42 milliseconds. ADIY and ASIY are roughly $40 \%$ to $70 \%$ costlier than ADIN and ASIN. PDIY and PSIY are the slowest, reaching 4.5 seconds at 1461 insertions. Per record, incremental methods level off at 365, whereas full block approaches continue to decline at a rate greater than 57\% at 1461 .

Regarding scaling (Figure 20), ASIN, ASIY, PSIN and PSIY are constant time. ASIN and PSIN take 0.6, ASIY 1.1, and PSIY 3.1 milliseconds per record. The rest are affected in various ways by existing records. The quickest configurations are ADFN, ADFY, ASFN, ASFY, PDFN, and PSFN. By 2362, they are just shy of ASIN and PSIN. PDFY and PSFY are minimally affected by existing records, with times ranging from 0.3 to 1.1 milliseconds. PDIN is next at roughly 0.7 to 3.6 milliseconds. ADIN and ADIY increase sharply from 0.7 to 6.9 milliseconds and 1.1 to 9.1 milliseconds, respectively. PDIY, at 3.5 to 6.1 milliseconds, is cheaper than ADIY at 945 and ADIN around 1800 . 
Figure 19. Server processing time in seconds and milliseconds per record by the number of records inserted. ADFN: AES-encrypted data, dynamic keys, full block storage, no server-side encryption; ADFY: AES-encrypted data, dynamic keys, full block storage, server-side encryption; ADIN: AES-encrypted data, dynamic keys, incremental storage, no server-side encryption; ADIY: AES-encrypted data, dynamic keys, incremental storage, server-side encryption; AES: Advanced Encryption Standard; ASFN: AES-encrypted data, static keys, full block storage, no server-side encryption; ASFY: AES-encrypted data, static keys, full block storage, server-side encryption; ASIN: AES-encrypted data, static keys, incremental storage, no server-side encryption; ASIY: AES-encrypted data, static keys, incremental storage, server-side encryption; PDFN: PRE-encrypted data, dynamic keys, full block storage, no server-side encryption; PDFY: PRE-encrypted data, dynamic keys, full block storage, server-side encryption; PDIN: PRE-encrypted data, dynamic keys, incremental storage, no server-side encryption; PDIY: PRE-encrypted data, dynamic keys, incremental storage, server-side encryption; PRE: proxy re-encryption; PSFN: PRE-encrypted data, static keys, full block storage, no server-side encryption; PSFY: PRE-encrypted data, static keys, full block storage, server-side encryption; PSIN: PRE-encrypted data, static keys, incremental storage, no server-side encryption; PSIY: PRE-encrypted data, static keys, incremental storage, server-side encryption.

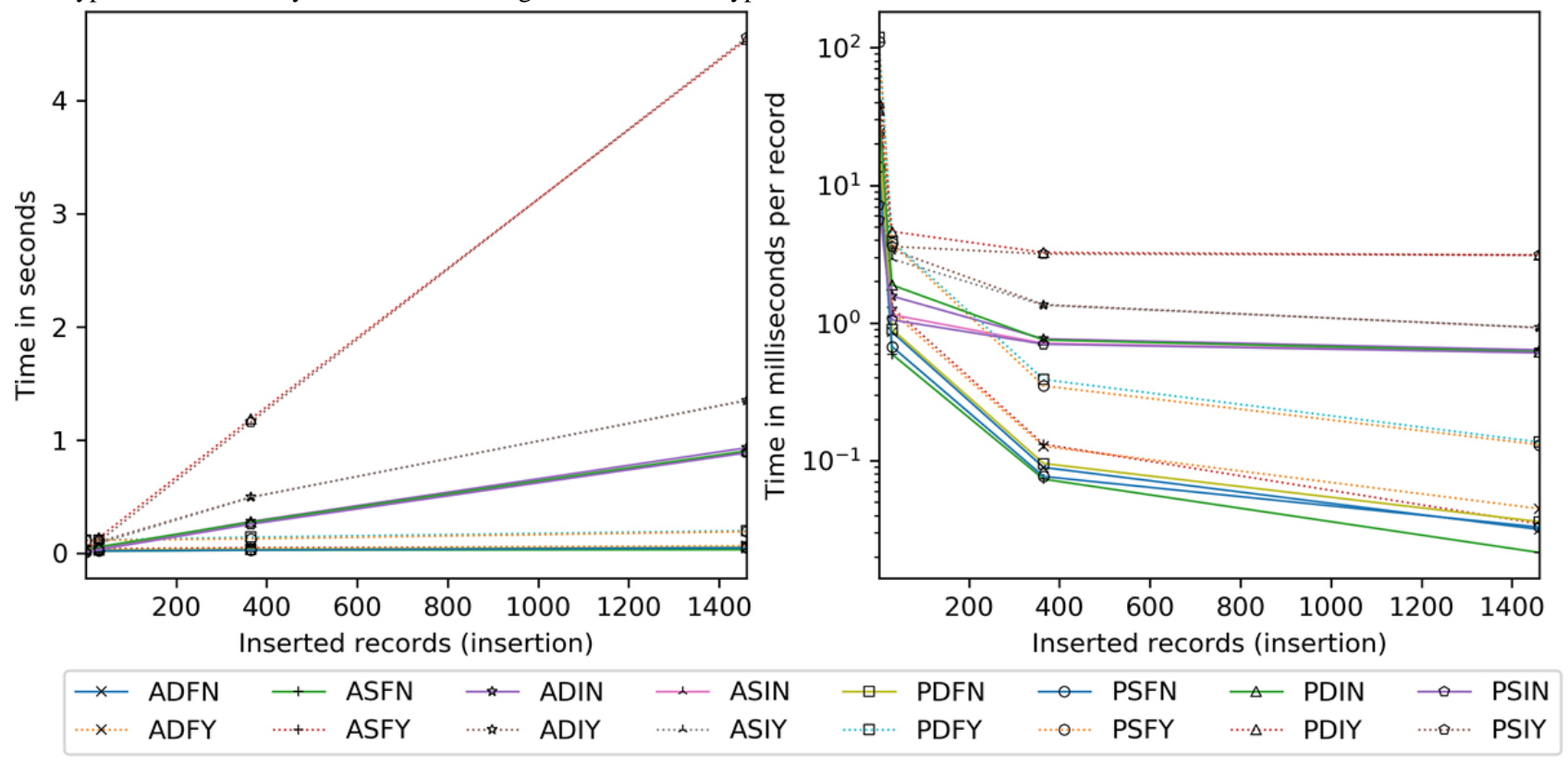


Figure 20. Server processing time in milliseconds per record added given an existing record set. ADFN: AES-encrypted data, dynamic keys, full block storage, no server-side encryption; ADFY: AES-encrypted data, dynamic keys, full block storage, server-side encryption; ADIN: AES-encrypted data, dynamic keys, incremental storage, no server-side encryption; ADIY: AES-encrypted data, dynamic keys, incremental storage, server-side encryption; AES: Advanced Encryption Standard; ASFN: AES-encrypted data, static keys, full block storage, no server-side encryption; ASFY: AES-encrypted data, static keys, full block storage, server-side encryption; ASIN: AES-encrypted data, static keys, incremental storage, no server-side encryption; ASIY: AES-encrypted data, static keys, incremental storage, server-side encryption; PDFN: PRE-encrypted data, dynamic keys, full block storage, no server-side encryption; PDFY: PRE-encrypted data, dynamic keys, full block storage, server-side encryption; PDIN: PRE-encrypted data, dynamic keys, incremental storage, no server-side encryption; PDIY: PRE-encrypted data, dynamic keys, incremental storage, server-side encryption; PRE: proxy re-encryption; PSFN: PRE-encrypted data, static keys, full block storage, no server-side encryption; PSFY: PRE-encrypted data, static keys, full block storage, server-side encryption; PSIN: PRE-encrypted data, static keys, incremental storage, no server-side encryption; PSIY: PRE-encrypted data, static keys, incremental storage, server-side encryption.

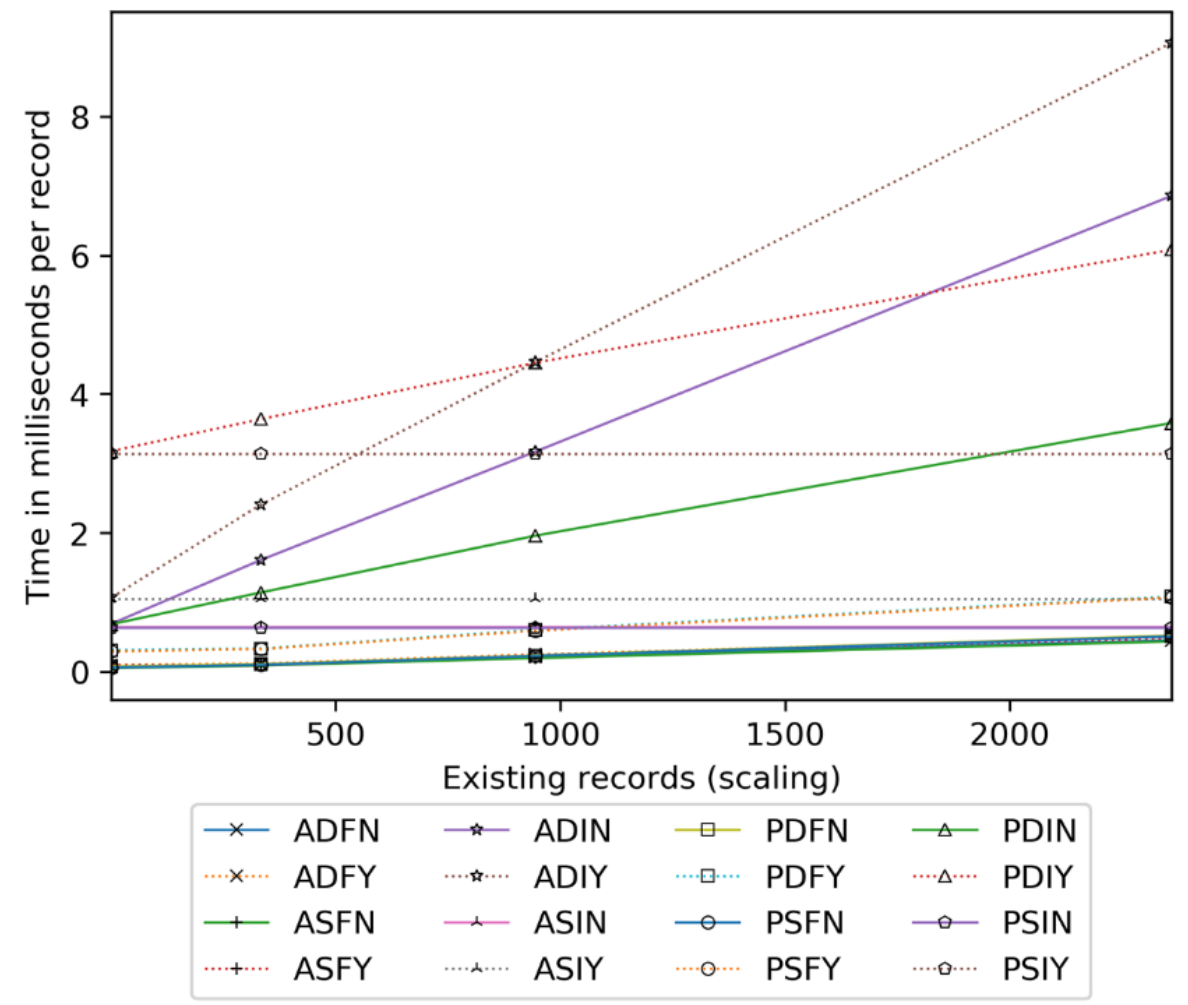

\section{Smart Contract Execution}

Smart contract execution by time and per record is assessed in this section, with results conveyed in Figure 21. Encryption key mode is not a factor in smart contract execution, as there is only 1 re-encryption key for all data; thus, it is not reported. As with the server-to-client network latency experiments, data include insertion and existing with 1461 additions sets.
In absolute terms, AFN, AFY, and AIN are the fastest, followed by PFN and PFY (progressing from $1 \%$ to $20 \%$ slower), AIY (23\%-183\%), PIN (46\%-854\%), and PIY (94\%-1679\%). Incremental server-side encryption is considerably expensive, doubling PRE and tripling AES times. Network latency accounts for around $9 \%$ of incremental and $19 \%$ of full block time. Per record, AIY, PIN, and PIY noticeably level-off by 365, and AFY, PFN, and PFY by 1461. AFN and AIN, however, descend beyond $3822(2361+1461)$ at $2 \%$. 
Figure 21. Smart contract execution time in seconds and milliseconds per record by the number of records processed. AES: Advanced Encryption Standard; AFY: AES-encrypted data, full block storage, server-side encryption; AFN: AES-encrypted data, full block storage, no server-side encryption; AIN: AES-encrypted data, incremental block storage, no server-side encryption; AIY: AES-encrypted data, incremental storage, server-side encryption; PFN: PRE-encrypted data, full block storage, no server-side encryption; PFY: PRE-encrypted data, full block storage, server-side encryption; PIN: PRE-encrypted data, incremental block storage, no server-side encryption; PIY: PRE-encrypted data, incremental storage, server-side encryption; PRE: proxy re-encryption.
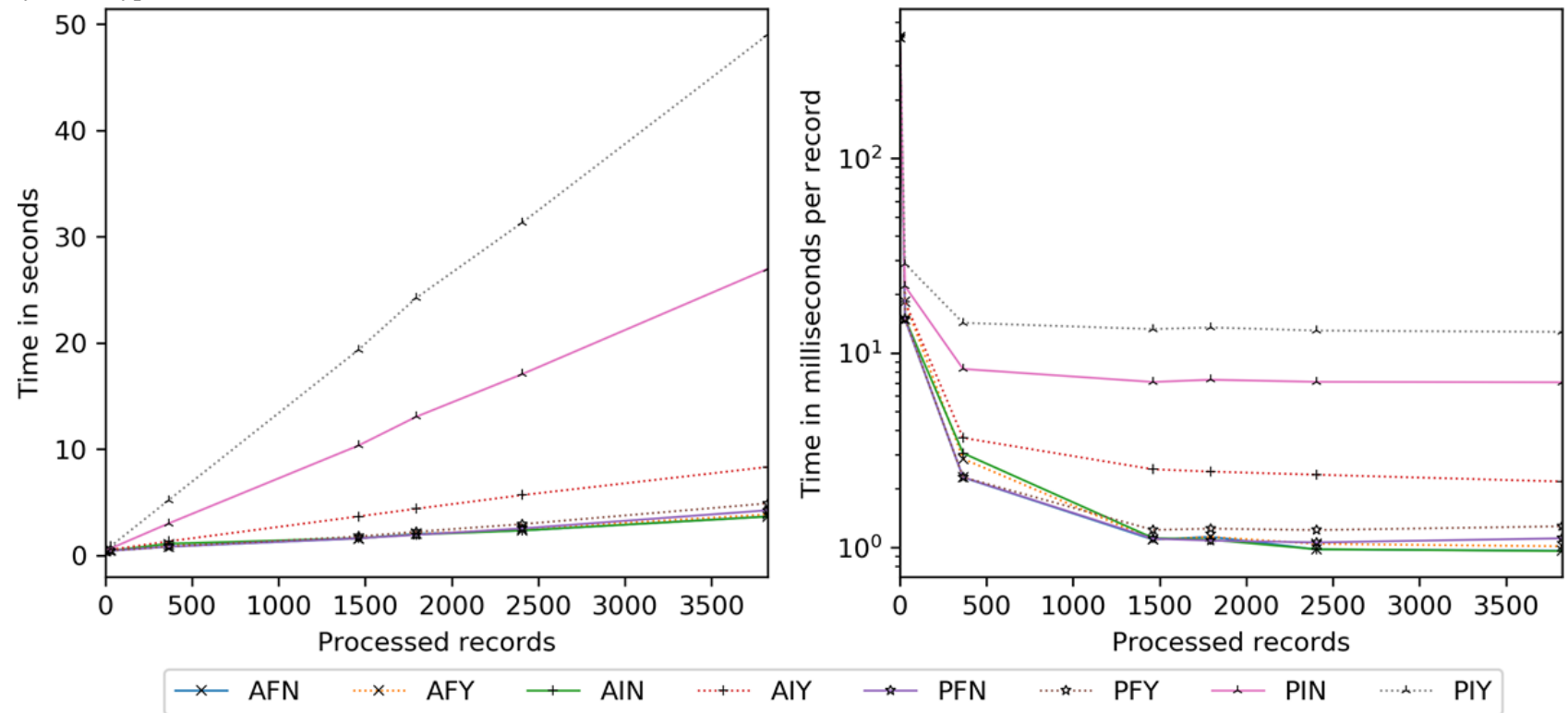

\section{Discussion}

\section{Principal Findings}

Figure 22 integrates pertinent results into a single visual for high-level performance analysis of client and server insertion (per record insertion cost given $n$ loaded records) and scaling (per record insertion cost given $n$ existing records) operations. Proceeding from top to bottom is client time in milliseconds (ie, section on Client Processing Time), server time in milliseconds (ie, section on Server Processing Time), and size in kilobytes (ie, section on Transmission Size).

The impracticality of full block approaches is apparent from the patient's vantage point. Whether inserting or scaling, block formation and transmission are resource intensive. For those with basic computers or mobile devices, or those operating on metered or low-bandwidth networks, these options should be avoided.

Catering to constrained environments are ASIN, PSIY, PDIN, PDIY, PSIN, and PSIY. ASIN, ASIY, PSIN, and PSIY are constant in bytes and server processing time, with decreasing client processing time because of network latency amortization over an otherwise constant process. PDIN and PDIY are constant in bytes and amortize latency as do the previous but irregular for the server (ie, server-side rekeying). Overall, ASIN and ASIY are the top performers. They require minimal time and bandwidth for record insertion, hold constant when scaling, and quickly execute smart contracts (refer to the section on Smart Contract Execution for details). The compromise is security. Static approaches, although fast, are susceptible to attacks (see the section on Smart Contracts and Multimedia Appendix 1).
Dynamic selections are more secure as data are continually rekeyed, preventing decryption by old or compromised keys. ADIN and ADIY are arguably the worst and operationally infeasible, as rekeying requires (1) the client to decrypt, encrypt, and transmit all information with each update and (2) the server to replace the existing block with the new, server-side-encrypted data.

PRE incremental methods are byte intensive because of excessive padding of the small experimental files and slow during smart contract execution. This is mostly mitigated through full block approaches. Unlike AES, the variability of PRE cipher size is vast. It has the potential to be compact and efficient or bloated and wasteful. PSIN and PSIY are subject to the same static key vulnerabilities as ASIN and ASIY and are more expensive. One must decide if cipher malleability justifies increased resource expenditure.

PDIN and PDIY are the only viable dynamic options. Server processing is insignificant for record insertions (a few milliseconds) but rises with scale. From 1 to 2361 , PDIN is $1 \%$ to $7 \%$ and PDIY $4 \%$ to $11 \%$, the magnitude of the client. Server processing is projected to eclipse client by around 40,000 and 37,000 records for PDIN and PDIY respectively. However, with appropriate hardware and in-memory databases, this cost can be reduced. Compared with ASIN and ASIY, both are marginally slower on the client, but roughly twice in bytes and latency.

Ultimately, several candidates emerge. ASIN and ASIY for speed, PSIN and PSIY for malleability, and PDIN and PDIY for malleability and security. Refer to Table 9 for a detailed comparison. 
Figure 22. Relative comparison of client and server processing time in milliseconds and transmission size in kilobytes per record by insertion and scaling. ADFN: AES-encrypted data, dynamic keys, full block storage, no server-side encryption; ADFY: AES-encrypted data, dynamic keys, full block storage, server-side encryption; ADIN: AES-encrypted data, dynamic keys, incremental storage, no server-side encryption; ADIY: AES-encrypted data, dynamic keys, incremental storage, server-side encryption; AES: Advanced Encryption Standard; ASFN: AES-encrypted data, static keys, full block storage, no server-side encryption; ASFY: AES-encrypted data, static keys, full block storage, server-side encryption; ASIN: AES-encrypted data, static keys, incremental storage, no server-side encryption; ASIY: AES-encrypted data, static keys, incremental storage, server-side encryption; PDFN: PRE-encrypted data, dynamic keys, full block storage, no server-side encryption; PDFY: PRE-encrypted data, dynamic keys, full block storage, server-side encryption; PDIN: PRE-encrypted data, dynamic keys, incremental storage, no server-side encryption; PDIY: PRE-encrypted data, dynamic keys, incremental storage, server-side encryption; PRE: proxy re-encryption; PSFN: PRE-encrypted data, static keys, full block storage, no server-side encryption; PSFY: PRE-encrypted data, static keys, full block storage, server-side encryption; PSIN: PRE-encrypted data, static keys, incremental storage, no server-side encryption; PSIY: PRE-encrypted data, static keys, incremental storage, server-side encryption.
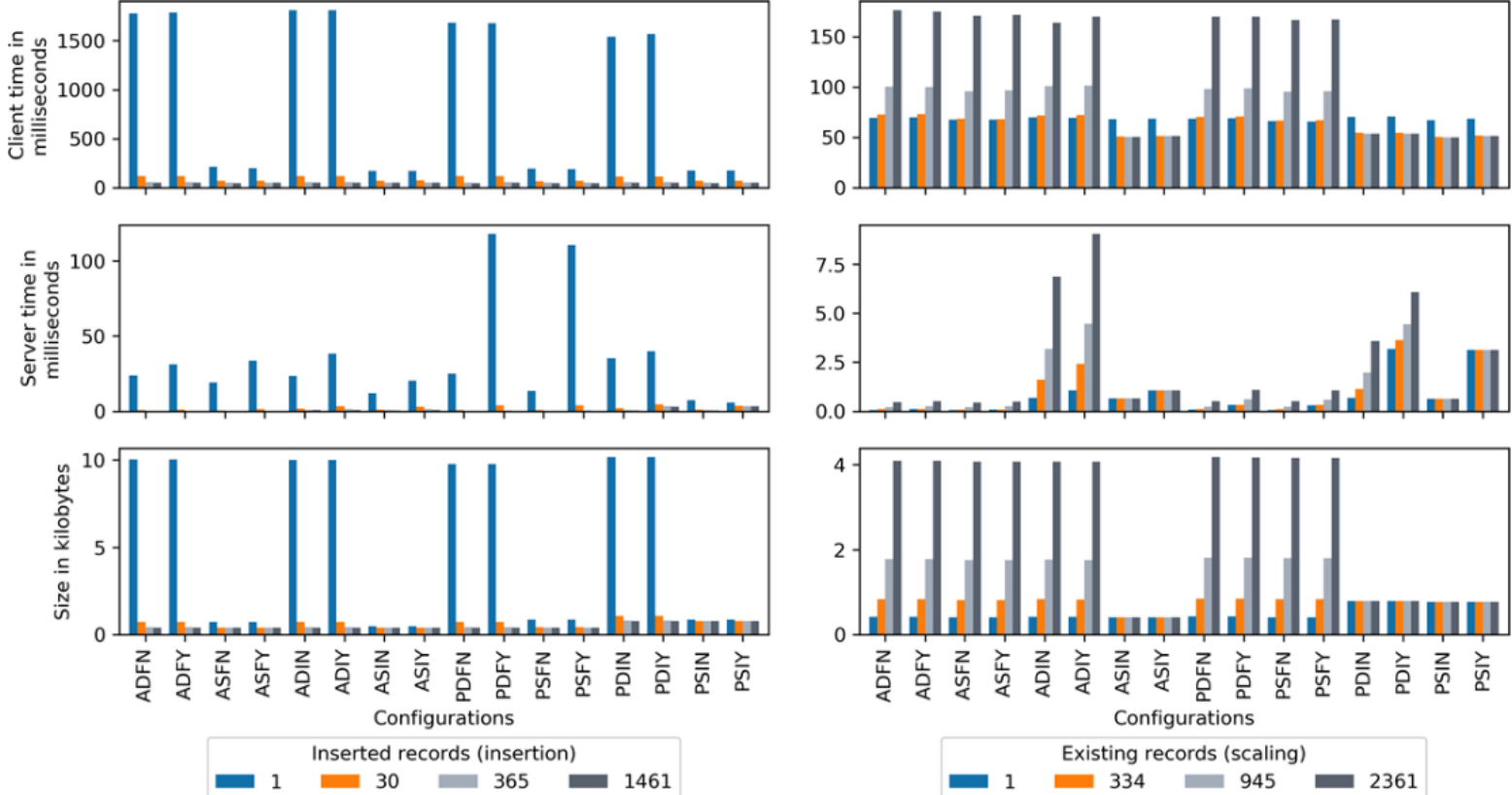

Table 9. Comparison of practical configurations by cipher malleability; security; insertion, scaling, and smart contract execution time; and transmitted bytes.

\begin{tabular}{|c|c|c|c|c|c|c|}
\hline Property & $\operatorname{ASIN}^{\mathrm{a}}$ & $\mathrm{ASIY}^{\mathrm{b}}$ & PSIN $^{\mathrm{c}}$ & PSIY $^{\mathrm{d}}$ & PDIN $^{\mathrm{e}}$ & PDIY $^{\mathrm{f}}$ \\
\hline Cipher malleability & No & No & Yes & Yes & Yes & Yes \\
\hline Security—dynamic keying & No & No & No & No & Yes & Yes \\
\hline Security—server-side encryption & No & Yes & No & Yes & No & Yes \\
\hline Client insertions time & Fastest & Fastest & Fast & Fast & Fast if $>30$ & Fast if $>30$ \\
\hline Client insertions bytes & Smallest & Smallest & Largest & Largest & Largest & Largest \\
\hline Server insertions time & Fast & Slow & Slowest & Slow & Fast & Slowest \\
\hline Client scaling time & Fastest, constant ${ }^{\mathrm{g}}$ & Fastest, constant ${ }^{\mathrm{g}}$ & Fastest, constant ${ }^{\mathrm{g}}$ & Fastest, constant ${ }^{\mathrm{g}}$ & Fast, constant ${ }^{\mathrm{g}}$ & Fast, constant ${ }^{\mathrm{g}}$ \\
\hline Client scaling bytes & $\begin{array}{l}\text { Smallest, con- } \\
\text { stant }\end{array}$ & $\begin{array}{l}\text { Smallest, con- } \\
\text { stant }\end{array}$ & Small, constant & Small, constant & Small, constant & Small, constant \\
\hline Server scaling time & Fast, constant & Slow, constant & Fast, constant & Slower, constant & Slower & Very slow \\
\hline Smart contract execution & Fastest & Fastest & Very slow & Very slow & Slowest & Slowest \\
\hline
\end{tabular}

${ }^{a}$ ASIN: Advanced Encryption Standard-encrypted data, static keys, incremental storage, no server-side encryption.

${ }^{\mathrm{b}}$ ASIY: Advanced Encryption Standard-encrypted data, static keys, incremental storage, server-side encryption.

${ }^{c}$ PSIN: proxy re-encryption-encrypted data, static keys, incremental storage, no server-side encryption.

${ }^{\mathrm{d}}$ PSIY: proxy re-encryption-encrypted data, static keys, incremental storage, server-side encryption.

${ }^{\mathrm{e}} \mathrm{PDIN}$ : proxy re-encryption-encrypted data, dynamic keys, incremental storage, no server-side encryption.

${ }^{f}$ PDIY: proxy re-encryption-encrypted data, dynamic keys, incremental storage, server-side encryption.

${ }^{\mathrm{g}}$ Constant time if latency, which is amortized over records, is not factored. 


\section{Limitations}

Our study has the following limitations. First, the results are consistent with AES-128 and EC-256 alone. It is impossible to extrapolate the effects a change may have. Second, the small experimental files resulted in excess PRE cipher padding. Although the records were legitimate, EHR data may produce different results. Third, only 1 smart contract, which dynamic options regenerate during an update, was present for testing. With many contracts and few existing records, overall performance may diminish. In addition, smart contract regeneration was not optimized as the entire contract was reproduced and transmitted instead of just the re-encryption keys. This modification has the potential to decrease the size by $41 \%$. Finally, server-side encryption only operates in dynamic mode. A static or periodic (eg, daily or after $x$ number of transactions) option would reduce server-side processing at the expense of security. This will especially benefit PRE schemes, as they suffer a tremendous penalty under the weight of rekeying data after each read.

\section{Conclusions and Future Work}

In this study, a proof-of-concept patient-centered blockchain-HealthChain-was presented. The posited framework promotes patient engagement and facilitates secure, mediated information exchange between patients and providers.
Redactable patient blocks, by way of chameleon hashing, were introduced to minimize data fragmentation, allow for in-place editing, and reduce resource consumption. PRE, smart contracts, and HL7 FHIR form the foundation of our proposed information exchange model, along with our 2PD PRE scheme and signature methods. A total of 16 experimental configurations were examined over 5 system dimensions by the cost of record insertion and scaling. Results indicate ASIN was the fastest and least bandwidth intensive, whereas PDIY was the best cryptographically, although the ultimate configuration rests with implementers and their desired level of speed and security.

Furthermore, 3 areas are targeted for future work. First, Barreto-Lynn-Scott [125] and Kachisa-Schaefer-Scott [126] EC families will be explored as potential replacements for the outdated jPBC curves. Second, as the proof-of-concept client tool is a deployed Web service on a client's machine (sufficient for testing), practical application necessitates an architectural redesign, wherein clients access HealthChain through a hosted, browser-based system. Hence, the cryptographic services will be ported to JavaScript for client-side execution, ensuring plaintext and generated secrets remain unexposed to nodes. Finally, we integrate our solution into Hyperledger Fabric to make use of their consensus, permissioning, and communications infrastructure.

\section{Conflicts of Interest}

None declared.

\section{Multimedia Appendix 1}

Proxy re-encryption mathematical foundation, proposed extensions, and proofs.

[PDF File (Adobe PDF File), 514KB-Multimedia Appendix 1]

\section{Multimedia Appendix 2}

Experimental configurations.

[PDF File (Adobe PDF File), 248KB-Multimedia Appendix 2]

\section{Multimedia Appendix 3}

Source code files.

[ZIP File (Zip Archive), 33MB-Multimedia Appendix 3]

\section{References}

1. Yaeger K, Martini M, Rasouli J, Costa A. Emerging blockchain technology solutions for modern healthcare infrastructure. J Sci Innov Med 2019;2(1):1. [doi: 10.29024/jsim.7]

2. Zhang P, White J, Schmidt DC, Lenz G, Rosenbloom ST. FHIRChain: applying blockchain to securely and scalably share clinical data. Comput Struct Biotechnol J 2018;16:267-278 [FREE Full text] [doi: 10.1016/j.csbj.2018.07.004] [Medline: 30108685]

3. Azaria A, Ekblaw A, Vieira T, Lippman A. MedRec: Using Blockchain for Medical Data Access and Permission Management. In: Proceedings of the 2nd International Conference on Open and Big Data. 2016 Presented at: OBD'16; August 22-24, 2016; Vienna, Austria. [doi: 10.1109/OBD.2016.11]

4. Library of Congress. 2016. Public Law 114-255-Dec 13, 2016 URL: https://www.congress.gov/114/plaws/publ255/ PLAW-114publ255.pdf [accessed 2019-02-01] [WebCite Cache ID 75rG0BbUq]

5. HealthIT. 2015. Report to Congress: Report on Health Information Blocking URL: https://www.healthit.gov/sites/default/ files/reports/info blocking 040915.pdf[WebCite Cache ID 75rGDwumh]

6. Bell L, Buchanan WJ, Cameron J, Lo O. Applications of blockchain within healthcare. Blockchain in Healthcare Today 2018 Jul 9;1:1-7. [doi: 10.30953/bhty.v1.8] 
7. Cyran MA. Blockchain as a foundation for sharing healthcare data. Blockchain in Healthcare Today 2018 Mar 23;1:1-6. [doi: 10.30953/bhty.v1.13]

8. Ribitzky R, St Clair J, Houlding DI, McFarlane CT, Ahier B, Gould M, et al. Pragmatic, interdisciplinary perspectives on blockchain and distributed ledger technology: paving the future for healthcare. Blockchain in Healthcare Today 2018 Mar 23;1:1-15. [doi: 10.30953/bhty.v1.24]

9. Yue X, Wang H, Jin D, Li M, Jiang W. Healthcare data gateways: found healthcare intelligence on blockchain with novel privacy risk control. J Med Syst 2016 Oct;40(10):218. [doi: 10.1007/s10916-016-0574-6] [Medline: 27565509]

10. Rabah K. Challenges \& opportunities for blockchain powered healthcare systems: a review. Mara Res J Med Health Sci 2017 Oct 16;1(1):45-52 [FREE Full text]

11. Xia Q, Sifah EB, Asamoah KO, Gao J, Du X, Guizani M. MeDShare: trust-less medical data sharing among cloud service providers via blockchain. IEEE Access 2017 Jul 24;5:14757-14767. [doi: 10.1109/ACCESS.2017.2730843]

12. Clauson KA, Breeden EA, Davidson C, Mackey TK. Leveraging blockchain technology to enhance supply chain management in healthcare:. Blockchain in Healthcare Today 2018 Mar 23;1:1-12. [doi: 10.30953/bhty.v1.20]

13. Mettler M. Blockchain Technology in Healthcare: The Revolution Starts Here. In: Proceedings of the 18th International Conference on e-Health Networking, Applications and Services. 2016 Presented at: Healthcom'16; September 14-16, 2016; Munich, Germany. [doi: 10.1109/HealthCom.2016.7749510]

14. Choudhury O, Sarker H, Rudolph N, Foreman M, Fay N, Dhuliawala M, et al. Enforcing human subject regulations using blockchain and smart contracts. Blockchain in Healthcare Today 2018 Mar 23;1:1-14. [doi: 10.30953/bhty.v1.10]

15. Esposito C, de Santis A, Tortora G, Chang H, Choo KR. Blockchain: a panacea for healthcare cloud-based data security and privacy? IEEE Cloud Comput 2018 Jan;5(1):31-37. [doi: 10.1109/MCC.2018.011791712]

16. Maslove DM, Klein J, Brohman K, Martin P. Using blockchain technology to manage clinical trials data: a proof-of-concept study. JMIR Med Inform 2018 Dec 21;6(4):e11949 [FREE Full text] [doi: 10.2196/11949] [Medline: 30578196]

17. Sylim P, Liu F, Marcelo A, Fontelo P. Blockchain technology for detecting falsified and substandard drugs in distribution: pharmaceutical supply chain intervention. JMIR Res Protoc 2018 Sep 13;7(9):e10163 [FREE Full text] [doi: 10.2196/10163] [Medline: 30213780]

18. Balsari S, Fortenko A, Blaya JA, Gropper A, Jayaram M, Matthan R, et al. Reimagining health data exchange: an application programming interface-enabled roadmap for India. J Med Internet Res 2018 Jul 13;20(7):e10725 [FREE Full text] [doi: 10.2196/10725] [Medline: 30006325$]$

19. Ichikawa D, Kashiyama M, Ueno T. Tamper-resistant mobile health using blockchain technology. JMIR Mhealth Uhealth 2017 Jul 26;5(7):e111 [FREE Full text] [doi: 10.2196/mhealth.7938] [Medline: 28747296]

20. Cisneros JL, Aarestrup FM, Lund O. Public health surveillance using decentralized technologies. Blockchain in Healthcare Today 2018 Mar 23;1:1-14. [doi: 10.30953/bhty.v1.17]

21. Dubovitskaya A, Xu Z, Ryu S, Schumacher M, Wang F. Secure and trustable electronic medical records sharing using blockchain. AMIA Annu Symp Proc 2017;2017:650-659 [FREE Full text] [Medline: 29854130]

22. Griggs KN, Ossipova O, Kohlios CP, Baccarini AN, Howson EA, Hayajneh T. Healthcare blockchain system using smart contracts for secure automated remote patient monitoring. J Med Syst 2018 Jun 6;42(7):130. [doi: 10.1007/s10916-018-0982-x] [Medline: 29876661]

23. Halamka JD, Lippman A, Ekblaw A. Harvard Business Review. 2017. The Potential for Blockchain to Transform Electronic Health Records URL: https://hbr.org/2017/03/the-potential-for-blockchain-to-transform-electronic-health-records

24. Ekblaw A, Azaria A, Halamka JD, Lippman A. A Case Study for Blockchain in Healthcare: 'Medrec' Prototype for Electronic Health Records and Medical Research Data. In: Proceedings of the 2nd International Conference on Open \& Big Data. 2016 Presented at: OBD'16; August 22-24, 2016; Vienna, Austria.

25. Roehrs A, da Costa CA, da Rosa RR. OmniPHR: a distributed architecture model to integrate personal health records. J Biomed Inform 2017 Jul;71:70-81 [FREE Full text] [doi: 10.1016/j.jbi.2017.05.012] [Medline: 28545835]

26. DeSalvo KB, Galvez D. HealthIT.gov. 2015. Connecting Health and Care for the Nation: A Shared Nationwide Interoperability Roadmap URL: https://www.healthit.gov/sites/default/files/comments_upload/

tascet comments to onc interoperability roadmap 201504 01.pdf [accessed 2019-02-01] [WebCite Cache ID 75rGOiVgd]

27. HL7 International. 2018. Fast Healthcare Interoperability Resources (FHIR) URL: https://www.hl7.org/fhir [accessed 2019-02-01] [WebCite Cache ID 75r90KGMd]

28. Kuo TT, Ohno-Machado L. arXiv. 2018. ModelChain: Decentralized Privacy-Preserving Healthcare Predictive Modeling Framework on Private Blockchain Networks URL: https://arxiv.org/abs/1802.01746

29. Kuo TT, Kim HE, Ohno-Machado L. Blockchain distributed ledger technologies for biomedical and health care applications. J Am Med Inform Assoc 2017 Nov 1;24(6):1211-1220 [FREE Full text] [doi: 10.1093/jamia/ocx068] [Medline: 29016974]

30. Tseng JH, Liao YC, Chong B, Liao SW. Governance on the drug supply chain via Gcoin blockchain. Int J Environ Res Public Health 2018 May 23;15(6):pii: E1055 [FREE Full text] [doi: 10.3390/ijerph15061055] [Medline: 29882861]

31. Radanović I, Likić R. Opportunities for use of blockchain technology in medicine. Appl Health Econ Health Policy 2018 Oct;16(5):583-590. [doi: 10.1007/s40258-018-0412-8] [Medline: $\underline{30022440]}$

32. Mackey TK, Nayyar G. A review of existing and emerging digital technologies to combat the global trade in fake medicines. Expert Opin Drug Saf 2017 May;16(5):587-602. [doi: 10.1080/14740338.2017.1313227] [Medline: 28349715] 
33. Roman-Belmonte JM, de la Corte-Rodriguez H, Rodriguez-Merchan EC. How blockchain technology can change medicine. Postgrad Med 2018 May;130(4):420-427. [doi: 10.1080/00325481.2018.1472996] [Medline: 29727247]

34. Benchoufi M, Ravaud P. Blockchain technology for improving clinical research quality. Trials 2017 Jul 19;18(1):335 [FREE Full text] [doi: 10.1186/s13063-017-2035-z] [Medline: 28724395]

35. Benchoufi M, Porcher R, Ravaud P. Blockchain protocols in clinical trials: transparency and traceability of consent. F1000Res 2017;6:66 [FREE Full text] [doi: 10.12688/f1000research.10531.5] [Medline: 29167732]

36. Cichosz SL, Stausholm MN, Kronborg T, Vestergaard P, Hejlesen O. How to use blockchain for diabetes health care data and access management: an operational concept. J Diabetes Sci Technol 2019 Mar;13(2):248-253 [FREE Full text] [doi: 10.1177/1932296818790281] [Medline: 30047789]

37. Till BM, Peters AW, Afshar S, Meara JG, Meara J. From blockchain technology to global health equity: can cryptocurrencies finance universal health coverage? BMJ Glob Health 2017;2(4):e000570 [FREE Full text] [doi: 10.1136/bmjgh-2017-000570] [Medline: 29177101]

38. Boulos MN, Wilson JT, Clauson KA. Geospatial blockchain: promises, challenges, and scenarios in health and healthcare. Int J Health Geogr 2018 Jul 5;17(1):25 [FREE Full text] [doi: 10.1186/s12942-018-0144-x] [Medline: 29973196]

39. Qu C, Tao M, Yuan R. A hypergraph-based blockchain model and application in internet of things-enabled smart homes. Sensors (Basel) 2018 Aug 24;18(9):pii: E2784 [FREE Full text] [doi: 10.3390/s18092784] [Medline: 30149523]

40. Zhou L, Wang L, Ai T, Sun Y. BeeKeeper 2.0: confidential blockchain-enabled IoT system with fully homomorphic computation. Sensors (Basel) 2018 Nov 05;18(11):pii: E3785 [FREE Full text] [doi: 10.3390/s18113785] [Medline: 30400673]

41. Zhu X, Badr Y. Identity management systems for the internet of things: a survey towards blockchain solutions. Sensors (Basel) 2018 Dec 1;18(12):pii: E4215 [FREE Full text] [doi: 10.3390/s18124215] [Medline: 30513733]

42. Park JS, Youn TY, Kim HB, Rhee KH, Shin SU. Smart contract-based review system for an IoT data marketplace. Sensors (Basel) 2018 Oct 22;18(10):pii: E3577 [FREE Full text] [doi: 10.3390/s18103577] [Medline: 30360413]

43. Dwivedi AD, Srivastava G, Dhar S, Singh R. A decentralized privacy-preserving healthcare blockchain for IoT. Sensors (Basel) 2019 Jan 15;19(2):pii: E326 [FREE Full text] [doi: 10.3390/s19020326] [Medline: 30650612]

44. Nakamoto S. Bitcoin.org. 2008. Bitcoin: A Peer-to-Peer Electronic Cash System URL: https://bitcoin.org/bitcoin.pdf]WebCite Cache ID 75m0ZCttp]

45. Bitcoin Forum. 2011. Proof of Stake Instead of Proof of Work URL: https://bitcointalk.org/index.php?topic=27787.0 [accessed 2019-02-01] [WebCite Cache ID 75r9zR885]

46. Buterin V, Griffith V. arXiv. 2017. Casper the Friendly Finality Gadget URL: https://arxiv.org/abs/1710.09437 [accessed 2019-02-01] [WebCite Cache ID 75rA6am4h]

47. Hyperledger - Open Source Blockchain Technologies. 2017. Introduction to Hyperledger Business Blockchain Design Philosophy and Consensus URL: https://www.hyperledger.org/wp-content/uploads/2017/08/

Hyperledger_Arch_WG_Paper_1_Consensus.pdf [accessed 2019-02-01] [WebCite Cache ID 75rA9MVgf]

48. Hyperledger Sawtooth. 2019. Introduction URL: https://sawtooth.hyperledger.org/docs/core/releases/latest/introduction. html [accessed 2019-02-01] [WebCite Cache ID 75rACSbao]

49. Hyperledger Fabric Docs. 2019. A Blockchain Platform for the Enterprise URL: https://hyperledger-fabric.readthedocs.io/ en/latest/ [accessed 2019-02-01] [WebCite Cache ID 75rAGGyjw]

50. Ateniese G, Magri B, Venturi D, Andrade E. Redactable Blockchain - or - Rewriting History in Bitcoin and Friends. In: Proceedings of the European Symposium on Security and Privacy. 2017 Presented at: EuroS\&P'17; April 26-28, 2017; Paris, France. [doi: 10.1109/EuroSP.2017.37]

51. Destefanis G, Marchesi M, Ortu M, Tonelli R, Bracciali A, Hierons R. Smart Contracts Vulnerabilities: A Call for Blockchain Software Engineering? In: Proceedings of the International Workshop on Blockchain Oriented Software Engineering. 2018 Presented at: IWBOSE'18; March 20, 2018; Campobasso, Italy. [doi: 10.1109/IWBOSE.2018.8327567]

52. Atzei N, Bartoletti M, Cimoli T. A Survey of Attacks on Ethereum Smart Contracts (SoK). In: Proceedings of the International Conference on Principles of Security and Trust. 2017 Presented at: POST'17; April 22-29, 2017; Uppsala, Sweden p. 164-186. [doi: 10.1007/978-3-662-54455-6 8]

53. Krawczyk H, Rabin T. CiteSeerX. 1997. Chameleon Hashing and Signatures URL: http://citeseerx.ist.psu.edu/viewdoc/ summary?doi=10.1.1.50.3262 [accessed 2019-02-01] [WebCite Cache ID 75rALtvwJ]

54. Krawczyk H, Rabin T. Chameleon Signatures. In: Proceedings of the Network and Distributed System Security Symposium. 2000 Presented at: NDSS'00; February 3-4, 2000; San Diego, California p. 143-154.

55. Krawczyk HM, Rabin TD. Google Patents. 2000. Chameleon Hashing and Signatures URL: https://patents.google.com/ patent/US6108783A/en

56. Luu L, Chu DH, Olickel H, Saxena P, Hobor A. Making Smart Contracts Smarter. In: Proceedings of the 2016 ACM SIGSAC Conference on Computer and Communications Security. 2016 Presented at: CCS'16; October 24-28, 2016; Vienna, Austria p. 254-269. [doi: 10.1145/2976749.2978309]

57. Delmolino K, Arnett M, Kosba A, Miller A, Shi E. Step by Step Towards Creating a Safe Smart Contract: Lessons and Insights from a Cryptocurrency Lab. In: Proceedings of the International Conference on Financial Cryptography and Data 
Security. 2016 Presented at: FC'16; February 22-26, 2016; Christ Church, Barbados p. 79-94. [doi: 10.1007/978-3-662-53357-4 6]

58. Kalra S, Goel S, Dhawan M, Sharma S. ZEUS: Analyzing Safety of Smart Contracts. In: Proceedings of the Network and Distributed Systems Security Symposium. 2018 Presented at: NDSS'18; February 18-21, 2018; San Diego, CA p. $18-21$.

59. Tsankov P, Dan A, Drachsler-Cohen D, Gervais A, Bünzli F, Vechev M. Securify: Practical Security Analysis of Smart Contracts. In: Proceedings of the 2018 ACM SIGSAC Conference on Computer and Communications Security. 2018 Presented at: CCS'18; October 15-19, 2018; Toronto, Canada p. 67-82. [doi: 10.1145/3243734.3243780]

60. Ateniese G, Fu K, Green M, Hohenberger S. Improved proxy re-encryption schemes with applications to secure distributed storage. ACM T Inform Syst Se 2006 Feb 1;9(1):1-30. [doi: 10.1145/1127345.1127346]

61. Mambo M, Okamoto E. Proxy cryptosystems: delegation of the power to decrypt ciphertexts. IEICE T Fund Electr Commun Comput Sci 1997;80(1):54-63.

62. Blaze M, Bleumer G, Strauss M. Divertible Protocols and Atomic Proxy Cryptography. In: Proceedings of the International Conference on the Theory and Applications of Cryptographic Techniques. 1998 Presented at: EUROCRYPT'98; May 31-June 4, 1998; Espoo, Finland p. 127-144. [doi: 10.1007/BFb0054122]

63. Nuñez D, Agudo I, Lopez J. Proxy re-encryption: analysis of constructions and its application to secure access delegation. J Netw Comput Appl 2017 Jun;87:193-209. [doi: 10.1016/j.jnca.2017.03.005]

64. Xu L, Wu X, Zhang X. CL-PRE: A Certificateless Proxy Re-Encryption Scheme for Secure Data Sharing with Public Cloud. In: Proceedings of the 7th ACM Symposium on Information, Computer and Communications Security. 2012 Presented at: ASIACCS'12; May 2-4, 2012; Seoul, Korea p. 87-88. [doi: 10.1145/2414456.2414507]

65. Ibraimi L, Tang Q, Hartel P, Jonker W. A Type-and-Identity-Based Proxy Re-encryption Scheme and Its Application in Healthcare. In: Proceedings of the Workshop on Secure Data Management. 2008 Presented at: SDM'08; August 24, 2008; Auckland, New Zealand p. 185-198. [doi: 10.1007/978-3-540-85259-9_12]

66. Lin H, Shao J, Zhang C, Fang Y. CAM: cloud-assisted privacy preserving mobile health monitoring. IEEE T Inf Foren Sec 2013 Jun 29;8(6):985-997. [doi: 10.1109/TIFS.2013.2255593]

67. Jia W, Zhu J, Cao Z, Wei L, Lin X. SDSM: A Secure Data Service Mechanism in Mobile Cloud Computing. In: Proceedings of the Conference on Computer Communications Workshops. 2011 Presented at: INFOCOM WKSHPS'11; April 10-15, 2011; Shanghai, China p. 1060-1065. [doi: 10.1109/INFCOMW.2011.5928784]

68. Thilakanathan D, Chen S, Nepal S, Calvo R, Alem L. A platform for secure monitoring and sharing of generic health data in the Cloud. Future Gener Comp Sy 2014 Jun;35:102-113. [doi: 10.1016/j.future.2013.09.011]

69. Qin Z, Xiong H, Wu S, Batamuliza J. A survey of proxy re-encryption for secure data sharing in Cloud computing. IEEE T Serv Comput 2017 Sep;13(9):1. [doi: 10.1109/TSC.2016.2551238]

70. Roy R, Mathai PP. Proxy re-encryption schemes for secure cloud data and applications: a survey. Int J Comput Appl 2017 Apr 17;164(5):1-6. [doi: 10.5120/ijca2017913642]

71. Kirshanova E. Proxy Re-Encryption from Lattices. In: Proceedings of the International Workshop on Public Key Cryptography. 2014 Presented at: PKC'14; March 26-28, 2014; Aires, Argentina p. 77-94. [doi: 10.1007/978-3-642-54631-0 5]

72. Zhang A, Bacchus A, Lin X. Consent-based access control for secure and privacy-preserving health information exchange. Secur Commun Netw 2016 Jul 7;9(16):3496-3508. [doi: 10.1002/sec.1556]

73. Au MH, Yuen TH, Liu JK, Susilo W, Huang X, Xiang Y, et al. A general framework for secure sharing of personal health records in cloud system. J Comput Syst Sci 2017 Dec;90:46-62. [doi: 10.1016/j.jcss.2017.03.002]

74. Wang XA, Ma J, Xhafa F, Zhang M, Luo X. Cost-effective secure e-health cloud system using identity based cryptographic techniques. Future Gener Comp Sy 2017 Feb;67:242-254. [doi: 10.1016/j.future.2016.08.008]

75. Huang Q, Wang L, Yang Y. Secure and privacy-preserving data sharing and collaboration in mobile healthcare social networks of smart cities. Secur Commun Netw 2017 Aug;2017:1-12. [doi: 10.1155/2017/6426495]

76. Syalim A, Nishide T, Sakurai K. Realizing Proxy Re-Encryption in the Symmetric World. In: Proceedings of the International Conference on Informatics Engineering and Information Science. 2011 Presented at: ICIEIS'11; November 14-16, 2011; Kuala Lumpur, Malaysia p. 259-274. [doi: 10.1007/978-3-642-25327-0 23]

77. Veeraragavan N, Rajesh MS. Proxy re-encryption using hybrid encryption scheme secure against chosen ciphertext attack. Int J Comput Sci Mobile Appl 2014 Jan;2(1):94-100 [FREE Full text]

78. Tysowski PK, Hasan MA. Hybrid attribute- and re-encryption-based key management for secure and scalable mobile applications in clouds. IEEE T Cloud Comput 2013 Jul;1(2):172-186. [doi: 10.1109/TCC.2013.11]

79. Nuñez D, Agudo I, Lopez J. Integrating OpenID With Proxy Re-Encryption to Enhance Privacy in Cloud-Based Identity Services. In: Proceedings of the International Conference on Cloud Computing Technology and Science. 2012 Presented at: ICCCS'12; December 3-6, 2012; Taipei, Taiwan. [doi: 10.1109/CloudCom.2012.6427551]

80. NuCypher. 2019. URL: https://www.nucypher.com/ [accessed 2019-02-01] [WebCite Cache ID 75rAoxjek]

81. National Institute of Standards and Technology. Federal Register. 2015. Federal Information Processing Standard (FIPS) 186-4, Digital Signature Standard; Request for Comments on the NIST-Recommended Elliptic Curves URL: https://www. federalregister.gov/documents/2015/10/20/2015-26539/ 
federal-information-processing-standard-fips-186-4-digital-signature-standard-request-for-comments [accessed 2019-02-01] [WebCite Cache ID 75rIZ8DIs]

82. Committee on National Security Systems. 2019. CNSS Policies URL: https://www.cnss.gov/CNSS/issuances/Policies.cfm [accessed 2019-02-01] [WebCite Cache ID 75rAuufT0]

83. Cryptome. 2015. Advisory Memorandum: Use of Public Standards for The Secure Sharing of Information Among National Security Systems URL: https://cryptome.org/2015/08/CNSS Advisory Memo 02-15.pdf [accessed 2019-02-01] [WebCite Cache ID 75rAySL9Y]

84. National Security Agency - Applications. 2018. CNSA Suite and Quantum Computing FAQ URL: https://apps.nsa.gov/ iaarchive/library/ia-guidance/ia-solutions-for-classified/algorithm-guidance/cnsa-suite-and-quantum-computing-faq.cfm [accessed 2019-02-01] [WebCite Cache ID 75rB1hWh7]

85. National Security Agency - Applications. Commercial National Security Algorithm Suite URL: https://apps.nsa.gov/ iaarchive/ [accessed 2019-02-01] [WebCite Cache ID 75rB8qqQW]

86. Joux A, Vitse V. Elliptic curve discrete logarithm problem over small degree extension fields. J Cryptol 2011 Nov 30;26(1):119-143. [doi: $10.1007 / \mathrm{s} 00145-011-9116-\mathrm{z}]$

87. Galbraith SD, Gaudry P. Recent progress on the elliptic curve discrete logarithm problem. Design Code Cryptogr 2015 Nov 23;78(1):51-72. [doi: 10.1007/s10623-015-0146-7]

88. Cao Z, Liu L. Cryptology ePrint Archive. 2015. On the Disadvantages of Pairing-Based Cryptography URL: https://eprint. iacr.org/2015/084.pdf

89. Nuñez D, Agudo I, Lopez J. NTRUReEncrypt: An Efficient Proxy Re-Encryption Scheme Based on NTRU. In: Proceedings of the 10th ACM Symposium on Information, Computer and Communications Security. 2015 Presented at: ASIA CCS'15; April 14-17, 2015; Singapore, Republic of Singapore p. 179-189. [doi: 10.1145/2714576.2714585]

90. Aono Y, Boyen X, Phong LT, Wang L. Key-Private Proxy Re-Encryption under LWE. In: Proceedings of the International Conference on Cryptology in India. 2013 Presented at: INDOCRYPT'13; December 7-10, 2013; Mumbai, India p. 1-18. [doi: $10.1007 / 978-3-319-03515-41]$

91. Xagawa K. Keita Xagawa. 2010. Cryptography with Lattices URL: http://xagawa.net/pdf/2010Thesis.pdf [accessed 2019-02-01] [WebCite Cache ID 75rBFweUG]

92. Xagawa K, Tanaka K. Kyoto University Research Information Repository. 2010. Proxy Re-Encryption Based on Learning With Errors URL: https://repository.kulib.kyoto-u.ac.jp/dspace/bitstream/2433/141576/1/1691-05.pdf

93. Kim KS, Jeong IR. Collusion-resistant unidirectional proxy re-encryption scheme from lattices. J Commun Netw 2016;18(1):1-7. [doi: 10.1109/JCN.2016.000003]

94. Lauter K. Postquantum opportunities: lattices, homomorphic encryption, and supersingular isogeny graphs. IEEE Security Privacy 2017;15(4):22-27. [doi: 10.1109/MSP.2017.3151338]

95. Martins P, Sousa L, Mariano A. A survey on fully homomorphic encryption: an engineering perspective. ACM Comput Surv 2018 Jan 12;50(6):1-33. [doi: 10.1145/3124441]

96. Tang PC, Ash JS, Bates DW, Overhage JM, Sands DZ. Personal health records: definitions, benefits, and strategies for overcoming barriers to adoption. J Am Med Inform Assoc 2006;13(2):121-126 [FREE Full text] [doi: 10.1197/jamia.M2025] [Medline: 16357345]

97. Agarwal R, Anderson C, Zarate J, Ward C. If we offer it, will they accept? Factors affecting patient use intentions of personal health records and secure messaging. J Med Internet Res 2013 Feb 26;15(2):e43 [FREE Full text] [doi: 10.2196/jmir.2243] [Medline: 23470453]

98. Al-Sahan A, Saddik B. Perceived challenges for adopting the personal health record (PHR) at Ministry of National Guard Health Affairs (MNGHA)- Riyadh. Online J Public Health Inform 2016;8(3):e205 [FREE Full text] [doi: 10.5210/ojphi.v8i3.6845] [Medline: 28210426]

99. HIMSS - Healthcare Information and Management Systems Society. 2008. Defining Key Health Information Technology Terms URL: https://www.himss.org/defining-key-health-information-technology-terms-onc-nahit[WebCite Cache ID $75 \mathrm{rDNMvNq}]$

100. HealthIT. 2019. Interoperability Standards: Advisory Office of the National Coordinator for Health IT URL: https://www. healthit.gov/isa/sites/isa/files/inline-files/2019ISAReferenceEdition.pdf [accessed 2019-02-01] [WebCite Cache ID 75rDJ5owA]

101. HealthIT. 2019. Interoperability Proving Ground URL: https://www.healthit.gov/techlab/ipg/[WebCite Cache ID 75rDPo3Go]

102. Apple. 2019. Empower Your Patients With Health Records on iPhone URL: https://www.apple.com/healthcare/health-records/ [accessed 2019-02-01] [WebCite Cache ID 75rDXPhy1]

103. Scarfone K, Souppaya M, Sexton M. NIST Computer Security Resource Center. 2007. Guide to Storage Encryption Technologies for End User Devices URL: https://csrc.nist.gov/publications/detail/sp/800-111/final[WebCite Cache ID 75rI4U6Zr]

104. Elgamal T. A public key cryptosystem and a signature scheme based on discrete logarithms. IEEE Trans Inf Theory 1985 Jul;31(4):469-472. [doi: 10.1109/TIT.1985.1057074]

105. Gentry C. Applied Cryptography Group | Stanford University. 2009. A Fully Homomorphic Encryption Scheme URL: https://crypto.stanford.edu/craig/craig-thesis.pdf 
106. Kogos KG, Filippova KS, Epishkina AV. Fully Homomorphic Encryption Schemes: The State of the Art. In: Proceedings of the Conference of Russian Young Researchers in Electrical and Electronic Engineering. 2017 Presented at: EIConRus'17; February 1-3, 2017; St Petersburg, Russia. [doi: 10.1109/EIConRus.2017.7910591]

107. El-Yahyaoui A, El Kettani MD. Evaluating AND Gates Over Encrypted Data in Cloud Computing. In: Proceedings of the International Conference on Advanced Communication Technologies and Networking. 2018 Presented at: CommNet'18; April 2-4, 2018; Marrakech, Morocco. [doi: 10.1109/COMMNET.2018.8360255]

108. EL-Yahyaoui A, El Kettani MD. A noise-free homomorphic evaluation of the AES circuits to optimize secure big data storage in cloud computing. In: Mohamed BA, Mohamed AA, editors. Innovations in Smart Cities and Applications: Proceedings of the 2nd Mediterranean Symposium on Smart City Applications. New York City: Springer; 2017:420-431.

109. Alves PG, Aranha DF. A framework for searching encrypted databases. J Internet Serv Appl 2018 Jan 3;9(1):1-18. [doi: 10.1186/s13174-017-0073-0]

110. Silva LV, Barbosa P, Marinho R, Brito A. Security and privacy aware data aggregation on cloud computing. J Internet Serv Appl 2018 Apr 2;9(1):1-13. [doi: 10.1186/s13174-018-0078-3]

111. Gentry CB. Google Patents. 2015. Fully Homomorphic Encryption URL: https://patents.google.com/patent/US9083526B2/ $\underline{\text { en }}$

112. Barker E. NIST Computer Security Resource Center. 2016. Recommendation for Key Management, Part 1: General URL: https://csrc.nist.gov/publications/detail/sp/800-57-part-1/rev-4/final [accessed 2019-02-01] [WebCite Cache ID 75rIBSia5]

113. de Caro A, Iovino V. jPBC: Java Pairing Based Cryptography. In: Proceedings of the Symposium on Computers and Communications. 2011 Presented at: ISCC'11; June 28-July 1, 2011; Kerkyra, Greece. [doi: 10.1109/ISCC.2011.5983948]

114. El Mrabet N, Joye M, editors. Guide to Pairing-Based Cryptography. First Edition. Boca Raton, Florida: Chapman and Hall/CRC; 2016.

115. Lynn B. Applied Cryptography Group|Stanford University. 2007. On the Implementation of Pairing-Based Cryptosystems URL: https://crypto.stanford.edu/pbc/thesis.pdf

116. Ateniese G, Benson K, Hohenberger S. Key-Private Proxy Re-Encryption. In: Proceedings of the Cryptographers’ Track at the RSA Conference. 2009 Presented at: CT-RSA'09; April 20-24, 2009; San Francisco, CA p. 279-294. [doi: 10.1007/978-3-642-00862-7 19]

117. Liu Q, Tan CC, Wu J, Wang G. Reliable Re-Encryption in Unreliable Clouds. In: Proceedings of the Global Telecommunications Conference. 2011 Presented at: GLOBECOM'11; December 5-9, 2011; Kathmandu, Nepal. [doi: 10.1109/GLOCOM.2011.6133609]

118. Sanka S, Hota C, Rajarajan M. Secure Data Access in Cloud Computing. In: Proceedings of the 4th International Conference on Internet Multimedia Services Architecture and Application. 2010 Presented at: IMSAA'10; December 15-17, 2010; Bangalore, India. [doi: 10.1109/IMSAA.2010.5729397]

119. Hota C, Sanka S, Rajarajan M, Nair SK. Capability-based cryptographic data access control in cloud computing. Int J Adv Netw Appl 2011;3(3):1152-1161 [FREE Full text]

120. Hylock R, Harris ST. Semantic Scholar. 2017. Healthcare Database Management for Health Informatics and Information Management Students: Challenges and Instruction Strategies-Part 2 URL: https://pdfs.semanticscholar.org/0622/ 7deb64113cbc81fda84889201f4eceb71e63.pdf?_ga=2.176924453.1425319861.1564981021-1326934932.1560835791

121. Speedtest by Ookla. 2019. URL: https://www.speedtest.net/ [accessed 2019-02-01] [WebCite Cache ID 75rE1UoI9]

122. HAPI FHIR. 2019. URL: http://hapifhir.io/ [accessed 2019-02-01] [WebCite Cache ID 75rDxeSTT]

123. Colorlib. 2019. Network Activities URL: https://colorlib.com/polygon/gentelella [accessed 2019-02-01] [WebCite Cache ID 75rDz4CP3]

124. Mandel JC, Kreda DA, Mandl KD, Kohane IS, Ramoni RB. SMART on FHIR: a standards-based, interoperable apps platform for electronic health records. J Am Med Inform Assoc 2016 Sep;23(5):899-908 [FREE Full text] [doi: 10.1093/jamia/ocv189] [Medline: 26911829]

125. Barreto PS, Lynn B, Scott M. Constructing Elliptic Curves with Prescribed Embedding Degrees. In: Proceedings of the International Conference on Security in Communication Networks. 2002 Presented at: SCN'02; September 11-13, 2002; Amalfi, Italy p. 257-267. [doi: 10.1007/3-540-36413-7 19]

126. Kachisa EJ, Schaefer EF, Scott M. Constructing Brezing-Weng Pairing-Friendly Elliptic Curves Using Elements in the Cyclotomic Field. In: Proceedings of the International Conference on Pairing-Based Cryptography. 2008 Presented at: Pairing'08; September 1-3, 2008; Egham, United Kingdom p. 126-135. [doi: 10.1007/978-3-540-85538-5 9]

\section{Abbreviations}

2PD: 2-party proxy re-encryption decryption

AES: Advanced Encryption Standard

ADFN: AES-encrypted data, dynamic keys, full block storage, no server-side encryption

ADI: AES-encrypted data, dynamic keys, incremental storage

ADIN: AES-encrypted data, dynamic keys, incremental storage, no server-side encryption

ADIY: AES-encrypted data, dynamic keys, incremental storage, server-side encryption 
AF: AES-encrypted data, full block storage

AFN: AES-encrypted data, full block storage, no server-side encryption

AIN: AES-encrypted data, incremental block storage, no server-side encryption

AIY: AES-encrypted data, incremental storage, server-side encryption

ASI: AES-encrypted data, static keys, incremental storage

ASIN: AES-encrypted data, static keys, incremental storage, no server-side encryption

ASIY: AES-encrypted data, static keys, incremental storage, server-side encryption

CFR: Code of Federal Regulations

EC: elliptic curves

ECC: elliptic curve cryptography

EHR: electronic health record

FHIR: Fast Healthcare Interoperability Resources

FIPS: Federal Information Processing Standard

HIPAA: Health Insurance Portability and Accountability Act of 1996

jPBC: Java Pairing-Based Cryptography library

Mbps: megabits per second

ONC: The Office of the National Coordinator for Health Information Technology

PCCH: Public-coin chameleon hash

PDF: PRE-encrypted data, dynamic encryption key full block storage

PDI: PRE-encrypted data, dynamic keys, incremental storage

PDIN: PRE-encrypted data, dynamic keys, incremental storage, no server-side encryption

PDIY: PRE-encrypted data, dynamic keys, incremental storage, server-side encryption

PF: PRE-encrypted data, full block storage

PFN: PRE-encrypted data, full block storage, no server-side encryption

PHR: personal health record

PI: PRE-encrypted data, incremental storage

PIN: PRE-encrypted data, incremental block storage, no server-side encryption

PIY: PRE-encrypted data, incremental storage, server-side encryption

PRE: proxy re-encryption

PSF: PRE-encrypted data, static keys, full block storage

PSI: PRE-encrypted data, static keys, incremental storage

PSIN: PRE-encrypted data, static keys, incremental storage, no server-side encryption

PSIY: PRE-encrypted data, static keys, incremental storage, server-side encryption

SHA: Secure Hash Algorithm

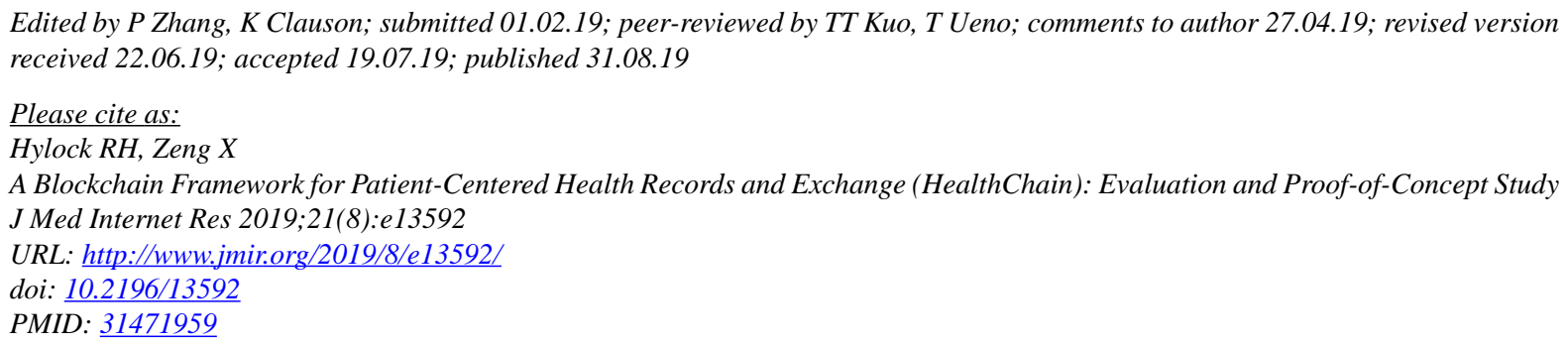

CRay Hales Hylock, Xiaoming Zeng. Originally published in the Journal of Medical Internet Research (http://www.jmir.org), 31.08.2019. This is an open-access article distributed under the terms of the Creative Commons Attribution License (https://creativecommons.org/licenses/by/4.0/), which permits unrestricted use, distribution, and reproduction in any medium, provided the original work, first published in the Journal of Medical Internet Research, is properly cited. The complete bibliographic information, a link to the original publication on http://www.jmir.org/, as well as this copyright and license information must be included. 